\title{
Inflationary spectral tilts as a result of the dilatation symmetry breaking
}

\author{
Pisin Chen, ${ }^{1,2,3}$ Jiro Matsumoto, ${ }^{1}$ and Rio Saitou ${ }^{1, *}$ \\ ${ }^{1}$ Leung Center for Cosmology and Particle Astrophysics, National Taiwan University, \\ Taipei, Taiwan 10617 \\ ${ }^{2}$ Department of Physics and Center for Theoretical Sciences, National Taiwan University, \\ Taipei, Taiwan 10617 \\ ${ }^{3}$ Kavli Institute for Particle Astrophysics and Cosmology, SLAC National Accelerator Laboratory, \\ Stanford University, Stanford, California 94305, USA
}

(Received 2 January 2019; published 24 July 2019)

\begin{abstract}
We derive the spectral indices and their runnings of single inflation models by a new approach. We perform a dilatation transformation to the linear cosmological perturbations and derive a current (non) conservation law. Using it, we construct a dilatation charge and a Ward-Takahashi identity for the two-point correlators, and derive two exact expressions for the tree-level spectral indices. First, we apply the slow roll expansion to one of the exact expressions. We calculate the spectral indices and their runnings up to the second and the third order of slow roll parameters, respectively, with use of the "horizon crossing formalism." By construction, our results are more rigorous and generic than the previous works. Then, we analyze another exact expression to understand how the perturbations and the slow roll parameters contribute to the spectral indices. By a numerical calculation, we confirm that only the behaviors of the slow roll parameters during a few e-folds around the horizon crossing affect significantly to the values of spectral indices. The analysis in this article indicates that if one cannot use the slow roll parameters, regardless of their values, as the expansion parameters around the horizon crossing, then one can no longer apply the slow roll expansion to the spectral indices, and it is thus necessary to apply the more generic method introduced here.
\end{abstract}

DOI: 10.1103/PhysRevD.100.023534

\section{INTRODUCTION}

Inflation is the phase of accelerated expansion in the early universe [1-3]. It can resolve the three big problems of the big bang universe and can create the seed of the large scale structure by the quantum fluctuation around the quasi-de Sitter background. Inflation is supposed to end and transit to the big bang universe accompanied with the reheating process. The observation of cosmic microwave background (CMB) anisotropy $[4,5]$ indicates that the temperature fluctuations of CMB are essentially Gaussian, which is in concordance with the quantum fluctuations predicted by the inflationary theory [6-9]. Thus, at the present time, inflation is considered as the standard theory of the early universe.

The observations of CMB anisotropy have imposed significant constraints to the numerous inflationary models. Initiated by the COBE project [10], WMAP [11], PLANCK

\footnotetext{
rsaito@ntu.edu.tw
}

Published by the American Physical Society under the terms of the Creative Commons Attribution 4.0 International license. Further distribution of this work must maintain attribution to the author(s) and the published article's title, journal citation, and DOI. Funded by SCOAP ${ }^{3}$.
[4,5], Keck/BICEP [12] projects have clarified that the primordial E-mode power spectrum of the temperature fluctuations is red-tilted in the Fourier space, and there is only a tiny fraction of the B-mode power spectrum. Mainly by the PLANCK, the changing ratio of E-mode spectral tilt and the size of non-Gaussianities of E-mode correlations are also constrained. While some inflationary models have been excluded by these observational facts, some other models still survive. Not only the potential-driven inflation [13-16], but also the kinetically driven inflation [17-19] and their hybrid type models [20-22] can still accord with the present observational constraints. To further distinguish and exclude them, we need more precise and accurate observations. It is expected that the next generation projects, such as PRISM, Lite-BIRD, CMB-S4, AliCPT, would obtain much more precise constraints to the scalar spectral tilt, its running and the ratio of E-mode and B-mode spectra. This, in turn, requires us to derive more precise theoretical predictions for those observables.

From the theoretical side, the inflationary observables are derived mainly by using the slow roll parameters. If and only if the slow roll parameters are small enough during the inflation, we can use them as expansion parameters for the observables. Among the observables, the spectral indices 
and their runnings have been derived up to the second order of the slow roll parameters [23-26]. The previous results are, however, restricted to the potential-driven inflation models only. Further, in [23], the authors imposed an ansatz for the model parameters to derive the power spectrum. In $[25,26]$, the authors performed an uncertain Taylor expansion for the power spectra, where the convergence of the expansion is not guaranteed. Moreover, their predictions rely on the slow roll expansion, so that we cannot apply them to a class of models where the slow roll parameters are not necessarily small throughout the inflation. In fact, many models are reported, in which the predictions of the slow roll expansion crucially deviates from the numerical calculation (e.g., [27-30]). Therefore, the previous methods according to the slow roll expansion are far from rigorous and generic.

On the other hand, there is another expansion method for the inflationary fluctuations, known as $\delta N$ formalism [31-34]. This method is based on the leading order gradient or momentum expansion parametrized by $\epsilon=k / a H$, where $k$ is the spatial momentum of the fluctuations, $a$ is the scale factor of a local homogeneous universe and $H$ is the Hubble parameter of the local universe. The formalism is reliable up to the first order of $\epsilon$, and it is applicable only for the fluctuations on the superhorizon scales where $\epsilon \ll 1$. Thus, we cannot use it for the models in which $O\left(\epsilon^{2}\right)$ terms induce significant effects to the fluctuations. Then, the gradient expansion has been recently extended up to the next-to-leading order for limited cases only [35]. Therefore, at the present, we cannot deal strictly with various models which can potentially survive the observational constraints. To face with the coming high precision observations, it is necessary to derive more rigorous and versatile theoretical predictions for the observables.

In this article, we derive the tree-level spectral indices exactly for single field inflation models by a new approach. By definition, the indices are intrinsically the scaling dimensions of the two-point correlators in the Fourier space. Hence, if we perform a dilatation transformation to the two-point correlators, we should naturally obtain exact expressions of the spectral indices. According to this idea, we define the dilatation transformation to the linear cosmological perturbation theory and construct the dilatation charge. Then, by using the Ward-Takahashi (WT) identity involved with the charge, we derive two expressions of the spectral indices for the scalar and the tensor perturbations without any ansatzes nor approximations. By construction, both expressions are exact and applicable to almost all wavelengths and all times for generic models. One of the two expressions is suitable for the slow roll expansion, while the other is suitable for numerical calculations. Since we ignore the quantum effects, our results remain at the classical level. It is still enough, however, to compare with the previous results for linear perturbations. We note that the dilatation transformation and the charge here are entirely different from those which are used to relate the three-point correlators with the two-point correlators [36-41]. In the previous works, the authors performed large gauge transformations that are included into the diffeomorphism, which were applied for the soft theorems. Here, we perform instead the dilatation transformation that is included into the conformal transformation regardless of the wavelengths of the perturbations.

This article is organized as follows. In Sec. II, firstly, we give generic actions for the linear perturbations. Next, we perform the dilatation transformation, and derive the current (non)conservation law and the charge. Then, constructing the WT identity, we derive the exact expressions for the spectral indices. In Sec. III, we expand the spectral indices by the slow roll parameters up to the second order. We derive rigorous and generic expressions for the spectral indices and their runnings, and compare the results with the previous ones. In Sec. IV, we analyze one of the exact expressions in detail and perform numerical calculations of the scalar spectral index for the Starobinsky model [2]. We compare the result with the slow roll expansion and the large $N_{*}$ expansion $[42,43]$. In the large $N_{*}$ expansion, we will expand the time-dependent parameters by the e-folding number at the horizon crossing time $N_{*}$. Section $\mathrm{V}$ is devoted to the summary. We add two Appendixes. In the Appendix A, we deform one of the exact expressions for the indices to the other exact expression. In Appendix B, we consider two specific models, power-law inflation [44] and ultra slow roll inflation [45], for the slow roll expansion. We use the unit $8 \pi G=c=\hbar=1$ throughout this article.

\section{WARD-TAKAHASHI IDENTITY}

We consider single field inflation models in a generic way. Instead of specifying a model, we just give a generic action $S\left[\phi, g_{\mu \nu}\right]$, which consists of the scalar field $\phi(x)$ and the metric field $g_{\mu \nu}(x)$. We assume that the given theory possesses the 4-dimensional diffeomorphism invariance and has the flat Friedmann-Lemaitre-Robertson-Walker background solution. For the single field inflation models, the background dynamics is governed by the homogeneous part of the scalar field $\phi(t)$. To consider the cosmological perturbation theory around the background, we use the Arnowitt-Deser-Misner formalism and take the comoving gauge as follows:

$$
\begin{aligned}
d s^{2} & =-N^{2} d t^{2}+{ }^{3} g_{i j}\left(d x^{i}+N^{i} d t\right)\left(d x^{j}+N^{j} d t\right), \\
{ }^{3} g_{i j} & =a^{2} \mathrm{e}^{2 \zeta}\left[\mathrm{e}^{\gamma}\right]_{i j}, \quad \partial_{j} \gamma_{i j}=0=\gamma_{i i}, \\
\phi & =\phi(t), \quad \delta \phi(x)=0 .
\end{aligned}
$$

$\zeta$ is the curvature perturbation and $\gamma_{i j}$ is the tracelesstransverse modes of tensor perturbation. In this gauge, the generic action reduces to 


$$
S\left[\phi, g_{\mu \nu}\right] \rightarrow S\left[t, K_{i j}, N,{ }^{3} g^{i j},{ }^{3} R_{i j}, \cdots\right]
$$

where $K_{i j}$ and ${ }^{3} R_{i j}$ are the extrinsic and intrinsic curvature, respectively, and the dots denote irrelevant higher derivative terms.

Following the derivation in $[46,47]$, we can naturally obtain the generic free actions of scalar perturbation and tensor perturbation:

$$
\begin{gathered}
S_{s}=\int d t d^{3} x a^{3}\left[\mathcal{G}_{s}(t) \dot{\zeta}^{2}-\frac{\mathcal{F}_{s}(t)}{a^{2}}(\nabla \zeta)^{2}\right], \\
S_{t}=\frac{1}{8} \int d t d^{3} x a^{3}\left[\mathcal{G}_{t}(t) \dot{\gamma}_{i j}^{2}-\frac{\mathcal{F}_{t}(t)}{a^{2}}\left(\nabla \gamma_{i j}\right)^{2}\right],
\end{gathered}
$$

where we restrict the perturbations to possess the Lorentzinvariant scaling. The dot denotes the partial derivative with respect to the cosmic time $t$. These generic free actions include all of Horndeski theory [48,49] and a part of degenerate-higher-order-scalar-tensor theories [50-54].

We define the speeds of sound, the conformal times, and the canonical perturbations for the scalar and the tensor perturbation, respectively as

$$
\begin{aligned}
c_{s}^{2}:=\frac{\mathcal{F}_{s}}{\mathcal{G}_{s}}, & \tau_{s}:=\int{ }^{t} \frac{c_{s}}{a} d t, \\
u_{s}(x):=z_{s} \zeta(x), & z_{s}=\sqrt{2} a\left(\mathcal{F}_{s} \mathcal{G}_{s}\right)^{1 / 4}, \\
c_{t}^{2}:=\frac{\mathcal{F}_{t}}{\mathcal{G}_{t}}, & \tau_{t}:=\int{ }^{t} \frac{c_{t}}{a} d t, \\
u_{t}(x) \mathbf{e}_{i j}:=z_{t} \gamma_{i j}(x), & z_{t}=\frac{a}{2}\left(\mathcal{F}_{t} \mathcal{G}_{t}\right)^{1 / 4} .
\end{aligned}
$$

Here, we defined the conformal times $\tau_{s, t}$ as the primitive functions of $c_{s, t}(t) / a(t)$, so that we set the constants of integration in Eqs. (5) and (7) to be zero. In the region where the integrands $c_{s, t}(t) / a(t)$ are monotonic functions of the cosmic time $t$, there exist one-to-one correspondences between $\tau_{s, t}$ and $t$, and we can use the conformal times instead of the cosmic time. $\mathbf{e}_{i j}$ is a polarization tensor satisfied with $\mathbf{e}_{i j} \mathbf{e}^{i j}=2$. Then, we rewrite the free action as

$$
\begin{gathered}
S_{s}=\int d \tau_{s} d^{3} x\left[\frac{1}{2}\left(\left(\partial_{\tau_{s}} u_{s}\right)^{2}-\left(\nabla u_{s}\right)^{2}\right)-\frac{1}{2} m_{s}^{2} u_{s}^{2}\right], \\
S_{t}=\int d \tau_{t} d^{3} x\left[\frac{1}{2}\left(\left(\partial_{\tau_{t}} u_{t}\right)^{2}-\left(\nabla u_{t}\right)^{2}\right)-\frac{1}{2} m_{t}^{2} u_{t}^{2}\right] \mathbf{e}^{i j} \mathbf{e}_{i j}
\end{gathered}
$$

where

$$
m_{s}^{2}=-\frac{\partial_{\tau_{s}}^{2} z_{s}}{z_{s}}, \quad m_{t}^{2}=-\frac{\partial_{\tau_{t}}^{2} z_{t}}{z_{t}}
$$

The free actions of canonical fields have the same forms, so that we can analyze their evolutions in the same manner.

To derive the spectral indices in the Fourier space, first, we consider a dilatation transformation defined in the coordinate space as

$x_{I}^{\mu} \rightarrow \tilde{x}_{I}^{\mu}=\mathrm{e}^{-\alpha} x_{I}^{\mu}, \quad u_{I}\left(x_{I}\right) \rightarrow \tilde{u}_{I}\left(\tilde{x}_{I}^{\mu}\right)=\mathrm{e}^{\alpha} u_{I}\left(x_{I}\right)$,

where the label $I=\{s, t\}, x_{I}^{\mu}=\left(\tau_{I}, \mathbf{x}\right)$ and $\alpha$ is a global constant parameter. Note that even if we redefine $\tau_{I}$ by shifting a finite constant from the present definitions (5) and (7), we can always obtain the same transformation as Eq. (12) by acting a time translation to the redefined $\tau_{I}$. Thus, the definition of $\tau_{I}$ never affects the results below. Under the dilatation transformation, the Lie derivatives of the field and the Lagrangian density become

$$
\begin{aligned}
\delta u_{I}\left(x_{I}\right) & =\left(1+x_{I}^{\mu} \partial_{I \mu}\right) u_{I}\left(x_{I}\right), \\
\delta \mathcal{L}_{I} & =\partial_{I \rho} X_{I}^{\rho}+\theta_{I} u_{I}^{2}, \\
X_{I}^{\mu} & :=x_{I}^{\mu} \mathcal{L}_{I}, \\
\theta_{I} & :=m_{I}^{2}+\frac{\tau_{I}}{2} \partial_{\tau_{I}} m_{I}^{2},
\end{aligned}
$$

where $\partial_{I \mu}:=\partial / \partial x_{I}^{\mu}$. We do not take a contraction for the label $I$. We define (non)conserved current as

$$
\begin{aligned}
j_{I}^{\mu} & :=\frac{\partial \mathcal{L}}{\partial\left(\partial_{\mu} u_{I}\left(x_{I}\right)\right)} \delta u_{I}-X_{I}^{\mu} \\
& =x_{I}^{\rho}\left(\partial_{I}^{\mu} u_{I} \partial_{I \rho} u_{I}-\delta_{\rho}^{\mu} \mathcal{L}_{I}\right)+\frac{1}{2} \partial_{I}^{\mu}\left(u_{I}^{2}\right)
\end{aligned}
$$

By using the Euler-Lagrange equation for $u_{I}\left(x_{I}\right)$, we can easily find a current (non)conservation law

$$
\partial_{I \mu} j_{I}^{\mu}=\theta_{I} u_{I}^{2}
$$

$\theta_{I}$ represents the breaking size of dilatation invariance of the free action for $u_{I}\left(x_{I}\right)$. By integrating Eq. (16) on the spacetime manifold, we can derive a relation between the dilatation charge $Q_{I}$ and the breaking term

$$
Q_{I}\left(\tau_{I}\right):=\int d^{3} x j_{I}^{0}\left(\tau_{I}, \mathbf{x}\right)
$$




$$
\begin{aligned}
Q_{I}\left(\tau_{I}\right)-Q_{I}\left(\tau_{I 0}\right) & =\int_{\tau_{I 0}}^{\tau_{I}} d \tilde{\tau}_{I} \partial_{\tilde{\tau}_{I}} \int d^{3} x j_{I}^{0}\left(\tilde{\tau}_{I}, \mathbf{x}\right) \\
& =\int_{\tau_{I 0}}^{\tau_{I}} d \tilde{\tau}_{I} \int d^{3} x \tilde{\partial}_{I \mu} j_{I}^{\mu}\left(\tilde{\tau}_{I}, \mathbf{x}\right) \\
& =\int_{\tau_{I 0}}^{\tau_{I}} d \tilde{\tau}_{I} \int d^{3} x \theta_{I} u_{I}^{2}\left(\tilde{\tau}_{I}, \mathbf{x}\right),
\end{aligned}
$$

where the spatial integration covers all of the spatial hypersurface. $\tau_{I 0}$ is an arbitrary time so that the $\tau_{I 0}$ dependence of the charge and of the last line of Eq. (18) will cancel out each other. This implies that we can take $\tau_{I 0}$ to $-\infty$ and extrapolate the behaviors of the perturbations and the background before the inflation to both sides of Eq. (18) even if we do not know the actual behavior of the whole of system before the inflation. The contribution from the extrapolated part will cancel each other out on both sides. We will extrapolate the field behaviors as in the Minkowski spacetime at the past infinity as usual.

Now, we canonically quantize the system. We define the conjugate momentum as

$$
\pi_{I}\left(x_{I}\right)=\frac{\partial \mathcal{L}_{I}}{\partial\left(\partial_{\tau_{I}} u_{I}\left(x_{I}\right)\right)}=\partial_{\tau_{I}} u_{I}\left(x_{I}\right)
$$

and introduce the canonical commutation relations at the same time

$$
\begin{gathered}
{\left[u_{I}\left(\tau_{I}, \mathbf{x}\right), \pi_{I}\left(\tau_{I}, \mathbf{y}\right)\right]=i \delta^{3}(\mathbf{x}-\mathbf{y}),} \\
{\left[u_{I}\left(\tau_{I}, \mathbf{x}\right), u_{I}\left(\tau_{I}, \mathbf{y}\right)\right]=\left[\pi_{I}\left(\tau_{I}, \mathbf{x}\right), \pi_{I}\left(\tau_{I}, \mathbf{y}\right)\right]=0 .}
\end{gathered}
$$

Then, we perform the Fourier expansion for the field

$u_{I}\left(x_{I}\right)=\int \frac{d k^{3}}{(2 \pi)^{3 / 2}}\left[a_{I \mathbf{k}} u_{I k}\left(\tau_{I}\right)+a_{I \mathbf{k}}^{\dagger} u_{I k}^{*}\left(\tau_{I}\right)\right] \mathrm{e}^{i \mathbf{k} \cdot(\mathbf{x}-\mathbf{y})}$,

where $k=|\mathbf{k}|$. The Heisenberg equation for $\pi_{I}$ require the mode function $u_{I k}$ to be satisfied with the MukhanovSasaki(MS) equation

$$
\partial_{\tau_{I}}^{2} u_{I k}+\left[k^{2}+m_{I}^{2}\left(\tau_{I}\right)\right] u_{I k}=0 .
$$

By imposing a normalization for the Wronskian $W$,

$$
W:=\left(\partial_{\tau_{I}} u_{I k}\right) u_{I k}^{*}-u_{I k}\left(\partial_{\tau_{I}} u_{I k}^{*}\right)=-i,
$$

we can normalize the commutation relations between the creation and annihilation operators as

$$
\left[a_{I \mathbf{k}}, a_{J \mathbf{q}}^{\dagger}\right]=\delta^{3}(\mathbf{k}-\mathbf{q}) \delta_{I J},
$$

$$
\left[a_{I \mathbf{k}}, a_{J \mathbf{q}}\right]=\left[a_{I \mathbf{k}}^{\dagger}, a_{J \mathbf{q}}^{\dagger}\right]=0
$$

We define the free vacuum $|0\rangle$ as

$$
a_{l \mathbf{k}}|0\rangle=0 \text { for }{ }^{\forall} \mathbf{k} \text {. }
$$

In Eq. (23), if the time-dependent mass term dominates over the oscillation term, namely, $k^{2} \ll\left|m_{I}^{2}\right|$, the mode function has the general asymptotic solutions

$$
u_{I k} \rightarrow A_{I}(k) z_{I}+B_{I}(k) z_{I} \int^{\tau_{I}} \frac{d \tilde{\tau_{I}}}{z_{I}^{2}}
$$

$A_{I}$ and $B_{I}$ are the normalization constants dependent on the spatial momentum $k$ only. We may determine them by requiring the free vacuum corresponds to the Bunch-Davies vacuum. We call the former solution $z_{I}$ and the latter solution $z_{I} \int{ }^{\tau_{I}} d \tilde{\tau}_{I} / z_{I}^{2}$ as the growing mode and the decaying mode, respectively.

Then, using Eq. (18), we write a WT identity for the two point function as

$$
\begin{aligned}
& \left\langle 0\left|\left[i Q_{I}\left(\tau_{I}\right), u_{I}\left(\tau_{I}, \mathbf{x}\right) u_{I}\left(\tau_{I}, \mathbf{y}\right)\right]\right| 0\right\rangle \\
& =\left\langle 0\left|\left[i Q_{I}(-\infty), u_{I}\left(\tau_{I}, \mathbf{x}\right) u_{I}\left(\tau_{I}, \mathbf{y}\right)\right]\right| 0\right\rangle \\
& \quad+\left\langle 0\left|\left[i \int_{-\infty}^{\tau_{I}} d \tilde{\tau}_{I} \int d^{3} z \theta_{I} u_{I}^{2}, u_{I}\left(\tau_{I}, \mathbf{x}\right) u_{I}\left(\tau_{I}, \mathbf{y}\right)\right]\right| 0\right\rangle,
\end{aligned}
$$

where we take $\tau_{I 0}=-\infty$. In general, of course, the above identity does not hold for an interacting field since the naive relation (18) will be changed by quantum corrections. However, at least for the free field, we can still use the classical one (18) as a relation between the quantum operators. We can calculate both sides of identity straightforwardly. The left-hand side becomes

$$
\begin{aligned}
& \left\langle 0\left|\left[i Q_{I}\left(\tau_{I}\right), u_{I}\left(\tau_{I}, \mathbf{x}\right) u_{I}\left(\tau_{I}, \mathbf{y}\right)\right]\right| 0\right\rangle \\
& =\int \frac{d^{3} k}{(2 \pi)^{3}}\left[-k \partial_{k}+\tau_{I} \partial_{\tau_{I}}-1\right]\left|u_{I k}\right|^{2} \mathrm{e}^{i \mathbf{k} \cdot(\mathbf{x}-\mathbf{y})} \\
& \quad+\int \frac{d k d \Omega}{(2 \pi)^{3}} \partial_{k}\left(k^{3}\left|u_{I k}\right|^{2} \mathrm{e}^{i \mathbf{k} \cdot(\mathbf{x}-\mathbf{y})}\right)
\end{aligned}
$$

where $d \Omega$ is a infinitesimal solid angle in the momentum space. On the other hand, after several integration by parts, the first term of the right-hand side becomes 


$$
\begin{aligned}
\left\langle 0\left|\left[i Q_{I}\left(\tau_{I 0}=-\infty\right), u_{I}\left(\tau_{I}, \mathbf{x}\right) u_{I}\left(\tau_{I}, \mathbf{y}\right)\right]\right| 0\right\rangle= & \int d^{3} k\left\{i \tau_{I 0}\left[\left(\partial_{\tau_{I}} u_{I k 0}\right)^{2} u_{k}^{* 2}-\left(\partial_{\tau_{I}} u_{I k 0}^{*}\right)^{2} u_{k}^{2}+\left(k^{2}+m_{I 0}^{2}\right)\left(u_{I k 0}^{2} u_{k}^{* 2}-u_{I k 0}^{* 2} u_{k}^{2}\right)\right]\right. \\
& -i\left(u_{I k 0} \partial_{\tau_{I}} u_{I k 0} u_{k}^{* 2}-u_{I k 0}^{*} \partial_{\tau_{I}} u_{I k 0}^{*} u_{k}^{2}\right)+\left(\partial_{\tau_{I}} u_{I k 0} k \partial_{k} u_{I k 0}-u_{I k 0} k \partial_{k} \cdot \partial_{\tau_{I}} u_{I k 0}\right) u_{k}^{* 2} \\
& \left.-\left(\partial_{\tau_{I}} u_{I k 0}^{*} k \partial_{k} u_{I k 0}^{*}-u_{I k 0}^{*} k \partial_{k} \cdot \partial_{\tau_{I}} u_{I k 0}^{*}\right) u_{k}^{2}\right\} \times \mathrm{e}^{i \mathbf{k} \cdot(\mathbf{x}-\mathbf{y})}
\end{aligned}
$$

where the quantities with the subscript 0 are evaluated at the time $\tau_{I 0}=-\infty$. We extrapolate the mode function as it obeys Eq. (23) even before the inflation. We also extrapolate the mass at the past infinity as it approaches to zero faster than $1 / \tau_{I}$ based on the estimation $m_{I}^{2} \sim-1 / \tau_{I}^{2}$. Then, we obtain

$$
\left.\tau_{I 0} m_{I 0}^{2}\right|_{\tau_{I 0}=-\infty}=0, \quad u_{I k 0}=\left.\frac{1}{\sqrt{2 k}} \mathrm{e}^{-i k \tau_{I}}\right|_{\tau_{I}=-\infty} .
$$

We note that these extrapolations do not need to follow the actual history of the universe before the inflation since the $\tau_{I 0}$ dependence will cancel each other on both sides of WT identity. It is sufficient to fix the mass and the mode function at past infinity, with which one can perform the calculation straightforwardly. Then, we find the first term in the right-hand side of Eq. (29) includes a surface term only:

$$
\left\langle 0\left|\left[i Q_{I}(-\infty), u_{I}\left(\tau_{I}, \mathbf{x}\right) u_{I}\left(\tau_{I}, \mathbf{y}\right)\right]\right| 0\right\rangle=i \int \frac{d k d \Omega}{(2 \pi)^{3}} \partial_{k}\left[\left(k^{3} \partial_{\tau_{I}} u_{I k 0} \cdot u_{I k 0} u_{I k}^{* 2}-\text { c.c. }\right) e^{i \mathbf{k} \cdot(\mathbf{x}-\mathbf{y})}\right]
$$

where c.c. implies the complex conjugate. The breaking term becomes

$$
\left\langle 0\left|\left[i \int_{-\infty}^{\tau_{I}} d \tilde{\tau}_{I} \int d^{3} z \theta_{I} u_{I}^{2}, u_{I}\left(\tau_{I}, \mathbf{x}\right) u_{I}\left(\tau_{I}, \mathbf{y}\right)\right]\right| 0\right\rangle=2 i \int \frac{d^{3} k}{(2 \pi)^{3}} \int_{-\infty}^{\tau_{I}} d \tilde{\tau}_{I} \theta_{I}\left(\tilde{\tau}_{I}\right)\left[u_{k}^{2}\left(\tilde{\tau}_{I}\right) u_{I k}^{* 2}\left(\tau_{I}\right)-u_{I k}^{* 2}\left(\tilde{\tau}_{I}\right) u_{I k}^{2}\left(\tau_{I}\right)\right] \mathrm{e}^{i \mathbf{k} \cdot(\mathbf{x}-\mathbf{y})} .
$$

Then, we consider the inverse Fourier transformation for the WT identity (29). Multiplying $\mathrm{e}^{-i(\mathbf{p} \cdot \mathbf{x}+\mathbf{q} \cdot \mathbf{y})}$ and taking integrations for $\mathbf{x}-$ and $\mathbf{y}-$ space, we obtain

$$
\begin{aligned}
& {\left[-p \partial_{p}+\tau_{I} \partial_{\tau_{I}}-1\right]\left|u_{I p}\right|^{2} \cdot(2 \pi)^{3} \delta^{3}(\mathbf{p}+\mathbf{q})+\int d k d \Omega \partial_{k}\left[(2 \pi)^{3} k^{3}\left|u_{I k}\right|^{2} \delta^{3}(\mathbf{k}-\mathbf{p}) \delta^{3}(\mathbf{k}+\mathbf{q})\right]} \\
& =\int d k d \Omega \partial_{k}\left[(2 \pi)^{3} k^{3}\left(\partial_{\tau_{I}} u_{I k 0} \cdot u_{I k 0} u_{I k}^{* 2}-\text { c.c. }\right) \cdot \delta^{3}(\mathbf{k}-\mathbf{p}) \delta^{3}(\mathbf{k}+\mathbf{q})\right] \\
& \quad+2 i \int_{-\infty}^{\tau_{I}} d \tilde{\tau}_{I} \theta_{I}\left(\tilde{\tau}_{I}\right)\left[u_{I p}^{2}\left(\tilde{\tau}_{I}\right) u_{I p}^{* 2}\left(\tau_{I}\right)-u_{I p}^{* 2}\left(\tilde{\tau}_{I}\right) u_{I p}^{2}\left(\tau_{I}\right)\right] \cdot(2 \pi)^{3} \delta^{3}(\mathbf{p}+\mathbf{q}) .
\end{aligned}
$$

For the surface terms (the second and the third terms), we consider two edge modes, $k=0, \infty$. From the structure of the Dirac's delta function, we find that the surface terms contribute only when $p=0, \infty$ and $\mathbf{p}+\mathbf{q}=0$, while the remaining terms exist as long as $\mathbf{p}+\mathbf{q}=0$. Therefore, besides $p=0$ and $\infty$ modes, the following relation must be satisfied:

$$
\left[p \partial_{p}-\tau_{I} \partial_{\tau_{I}}+1\right]\left|u_{I p}\right|^{2}=-2 i \int_{-\infty}^{\tau_{I}} d \tilde{\tau}_{I} \theta_{I}\left(\tilde{\tau}_{I}\right)\left[u_{I p}^{2}\left(\tilde{\tau}_{I}\right) u_{I p}^{* 2}\left(\tau_{I}\right)-u_{I p}^{* 2}\left(\tilde{\tau}_{I}\right) u_{I p}^{2}\left(\tau_{I}\right)\right] .
$$

We regard this relation as the WT identity in the momentum space, which can be applicable for all modes except for $p=0$ and $\infty$ modes. In the next section, we will see that the identity (36) perfectly reproduces the previous results of the spectral index for the canonical scalar field [23] without any ansatzes for the parameters. We comment also that a massless free scalar field on Minkowski spacetime or de Sitter spacetime is formally satisfied with the WT identity (36) even for $p=0$ and $\infty$ modes. To specify a rigorous treatment for the surface terms lies beyond the scope of this paper, but it might be connected with some symmetries of vacuum like the residual gauge symmetries [36-41]. We put this issue for future investigation. 
We define the tree level power spectra of the scalar perturbation and the tensor perturbation as

$$
\begin{aligned}
& P_{s}:=\frac{k^{3}}{2 \pi^{2}}\left|\frac{u_{s k}}{z_{s}}\right|^{2}, \\
& P_{t}:=2 \frac{k^{3}}{2 \pi^{2}}\left|\frac{u_{t k}}{z_{t}}\right|^{2} .
\end{aligned}
$$

The scalar and tensor spectral indices are defined as

$$
\begin{gathered}
n_{s}-1:=\frac{d \ln P_{s}}{d \ln k}=\frac{k \partial_{k} P_{s}}{P_{s}}, \\
n_{t}:=\frac{d \ln P_{t}}{d \ln k}=\frac{k \partial_{k} P_{t}}{P_{t}} .
\end{gathered}
$$

These relations imply

$$
\begin{aligned}
& k \partial_{k}\left|u_{s k}\right|^{2}=\left(n_{s}-4\right)\left|u_{s k}\right|^{2}, \\
& k \partial_{k}\left|u_{t k}\right|^{2}=\left(n_{t}-3\right)\left|u_{t k}\right|^{2} .
\end{aligned}
$$

To derive the explicit expressions of the spectral indices, we substitute Eqs. (40) and (41) to the WT identity (36). Then, we finally obtain

$$
\begin{gathered}
n_{I}=\mathcal{N}_{I}+2+2 \tau_{I} \frac{\partial_{\tau_{\tau}}\left|u_{I k}\right|}{\left|u_{I k}\right|}+\Theta_{I} \\
\Theta_{I}:=4 \operatorname{Im}\left[\frac{u_{I k}^{* 2}\left(\tau_{I}\right)}{\left|u_{I k}\left(\tau_{I}\right)\right|^{2}} \int_{-\infty}^{\tau_{I}} \theta_{I}\left(\tilde{\tau}_{I}\right) u_{I k}^{2}\left(\tilde{\tau}_{I}\right) d \tilde{\tau}_{I}\right] .
\end{gathered}
$$

where $\mathcal{N}_{s}=1, \mathcal{N}_{t}=0$. We can deform $n_{I}$ into another form which is useful for a numerical calculation. Performing several integrations by parts with use of Eqs. (23) and (24), we obtain

$$
\begin{gathered}
n_{I}=\mathcal{N}_{I}+1-4 \operatorname{Im}\left[\frac{\psi_{I k}^{* 2}}{\left|\psi_{I k}\right|^{2}} \int_{-\infty(1+i \varepsilon)}^{\tau_{I}} z_{I}^{2}\left(\partial_{\tilde{\tau}_{I}} \psi_{I k}\right)^{2} d \tilde{\tau}_{I}\right], \\
\psi_{I k}:=\frac{u_{I k}}{z_{I}},
\end{gathered}
$$

where we take the contour the same as the $i \varepsilon$ prescription. See Appendix A for the technical detail of this deformation.

Here, we stress that all of the above results (42)-(44) are exact expressions of the tree level spectral indices without any ansatzs nor approximations. Basically, we can use them for all time and all wavelengthes except for $k=0$ and $\infty$ modes, not restricted to the superhorizon scales. We can apply Eqs. (42)-(44) to all of the single field inflation models which possess the Lorentz-invariant scaling even if the background and the perturbations evolve in highly nontrivial ways.

\section{SLOW ROLL EXPANSION}

In this section, we expand the exact expression (42) by the slow roll parameters up to the second order and compare the results with the previous works. First, we introduce the e-folding number $n$ as

$$
\begin{aligned}
& n:=\ln \left(\frac{a}{a_{\mathrm{in}}}\right), \\
& a=a_{\mathrm{in}} \mathrm{e}^{n},
\end{aligned}
$$

where $a_{\text {in }}$ is an initial finite value of the scale factor at a certain time, and thus $n_{\text {in }}=0$. If the universe always expands, we can use $n$ as a clock. Next, we define the generic slow roll parameters following [48] as

$$
\begin{aligned}
\epsilon_{1} & :=-\frac{d \ln H}{d n}, & \epsilon_{i+1} & :=\frac{d \ln \epsilon_{\mathrm{i}}}{d n}, \\
f_{I 1} & :=\frac{d \ln \mathcal{F}_{I}}{d n}, & f_{I i+1} & :=\frac{d \ln f_{I i}}{d n}, \\
g_{I 1} & :=\frac{d \ln \mathcal{G}_{I}}{d n}, & g_{I i+1} & :=\frac{d \ln g_{I i}}{d n},
\end{aligned}
$$

where $H:=d \ln a / d t$ is the Hubble parameter and $\mathcal{F}_{I}$ and $\mathcal{G}_{I}$ are the coefficient functions appearing in the original free actions (3) and (4). By using $n$ and the slow roll parameters, we can expand the conformal time as

$$
\begin{aligned}
\tau_{I} & =\int^{n} \frac{c_{I}}{a H} d \tilde{n}=-\frac{c_{I}}{a H} \sum_{i=0}^{\infty} \delta_{I i}, \\
\delta_{I 0} & =1, \\
\delta_{I 1} & =\epsilon_{1}+\frac{1}{2}\left(f_{I 1}-g_{I 1}\right), \\
\delta_{I i} & :=\delta_{I 1} \delta_{I i-1}+\delta_{I i-1, n} \quad \text { for } i \geq 2 .
\end{aligned}
$$

where ", $n$ " denotes the differentiation with respect to $n$. The subscript $i$ on $\delta_{I i}$ corresponds to the order of slow roll parameters. Using them, up to the second order, the breaking size $\theta_{I}$ becomes

$$
\theta_{I}=\frac{3}{2 \tau_{I}^{2}}\left[\epsilon_{1} \epsilon_{2}+\frac{1}{4}\left(3 f_{I 1} f_{I 2}-g_{I 1} g_{I 2}\right)+O\left(\epsilon_{i}^{3}\right)\right] .
$$

We denoted higher order terms of the slow roll parameters than the second order by $O\left(\epsilon_{i}^{3}\right)$. To evaluate $\Theta_{I}(43)$ up to the second order of slow roll parameters, we expand the mode function also as follows:

$$
u_{I k}\left(\tau_{I}\right)=\sum_{i=0}^{\infty} u_{I k}^{(i)}\left(\tau_{I}\right)
$$


where $u_{I k}^{(i)}$ is the $i$ th order quantity of slow roll parameters. Since $\theta_{I}$ is already of the second order, we need the zeroth order term of the mode function $u_{I k}^{(0)}$ only. In the slow roll expansion, the zeroth order term of mode function in the quasi-de Sitter spacetime must be given by that in the exact de Sitter spacetime, so we can replace the mode function in $\Theta_{I}$ with

$$
u_{I k} \approx u_{I k}^{(0)}=\frac{-i}{\sqrt{2 k^{3}} \tau_{I}}\left(1+i k \tau_{I}\right) \mathrm{e}^{-i k \tau_{I}} .
$$

Substituting Eqs. (49) and (51) into Eq. (43), we obtain

$$
\begin{aligned}
\Theta_{I}= & -2\left[\epsilon_{1} \epsilon_{2}+\frac{1}{4}\left(3 f_{I 1} f_{I 2}-g_{I 1} g_{I 2}\right)\right] \\
& \times\left[C+\ln \left(-k \tau_{I}\right)+O\left(k \tau_{I}\right)\right]+O\left(\epsilon_{i}^{3}\right), \\
C:= & \gamma_{E}+\ln 2-2,
\end{aligned}
$$

where $\gamma_{E}$ is the Euler-Mascheroni constant and $C \approx$ -0.729637 . In the calculation, we treated the second order product of slow roll parameters as a constant since its variation becomes of the third order.

Now, we focus on the case where the asymptotic solutions of mode function (28) are governed by the growing mode ${ }^{1}$

$$
u_{I k} \rightarrow A_{I}(k) z_{I} \quad \text { when }\left|k \tau_{I}\right| \rightarrow 0 .
$$

In this case, $\psi_{I k}=u_{I k} / z_{I}$ and the spectra (37) depend on the spatial momentum only,

$\psi_{I k} \rightarrow A_{I}(k), \quad P_{s} \rightarrow \frac{k^{3}}{2 \pi^{2}}\left|A_{s}(k)\right|^{2}, \quad P_{t} \rightarrow \frac{k^{3}}{\pi^{2}}\left|A_{t}(k)\right|^{2}$,

which implies that they almost conserve on the superhorizon scales. The asymptotic values of spectral indices become

$$
\begin{aligned}
\left.n_{I}\right|_{\left|k \tau_{I}\right| \rightarrow 0}= & \mathcal{N}_{I}-2 \epsilon_{1}-\frac{1}{2}\left(3 f_{I 1}-g_{I 1}\right)-\left\{2 \epsilon_{1}^{2}+\frac{\epsilon_{1}}{2}\left(5 f_{I 1}-3 g_{I 1}\right)+\frac{1}{4}\left(f_{I 1}-g_{I 1}\right)\left(3 f_{I 1}-g_{I 1}\right)\right. \\
& \left.+(2 C+2) \epsilon_{1} \epsilon_{2}+\frac{3 C+2}{2} f_{I 1} f_{I 2}-\frac{C+2}{2} g_{I 1} g_{I 2}+\left[2 \epsilon_{1} \epsilon_{2}+\frac{1}{2}\left(3 f_{I 1} f_{I 2}-g_{I 1} g_{I 2}\right)\right] \ln \left(-k \tau_{I}\right)\right\}+O\left(\epsilon_{i}^{3}\right) .
\end{aligned}
$$

We note that although Eq. (55) seems to depend on the time, actually it does not since the asymptotic values of the spectra depend on the spatial momentum only. Therefore, we can change the time in Eq. (55) to an arbitrary time. If we change it to the horizon crossing time for the mode $k_{*}$,

$$
\left|\tau_{I}\right| \Rightarrow \frac{1}{a_{*} H_{*}}=\frac{1}{k_{*}}
$$

we finally obtain the asymptotic values of spectral indices in a generic form as

$$
\begin{aligned}
\left.n_{I}(k)\right|_{\left|k \tau_{I}\right| \rightarrow 0}= & \mathcal{N}_{I}-2 \epsilon_{1 *}-\frac{1}{2}\left(3 f_{I 1 *}-g_{I 1 *}\right)-\left\{2 \epsilon_{1 *}^{2}+\frac{\epsilon_{1 *}}{2}\left(5 f_{I 1 *}-3 g_{I 1 *}\right)+\frac{1}{4}\left(3 f_{I 1 *}^{2}-4 f_{I 1 *} g_{I 1 *}+g_{I 1 *}^{2}\right)+(2 C+2) \epsilon_{1 *} \epsilon_{2 *}\right. \\
& \left.+\frac{3 C+2}{2} f_{I 1 *} f_{I 2 *}-\frac{C+2}{2} g_{I 1 *} g_{I 2 *}+\left[2 \epsilon_{1 *} \epsilon_{2 *}+\frac{1}{2}\left(3 f_{I 1 *} f_{I 2 *}-g_{I 1 *} g_{I 2 *}\right)\right] \ln \left(\frac{k}{k_{*}}\right)\right\}+O\left(\epsilon_{i *}^{3}\right)
\end{aligned}
$$

All of the slow-roll parameters are evaluated at the horizon crossing time for the mode $k_{*}$ when $k_{*}=a_{*} H_{*}\left(n_{*}\right)$. The logarithmic term represents the deviation between the modes $k$ and $k_{*}$, and it disappears if we choose $k=k_{*}$. We can safely ignore the higher order terms denoted by $O\left(\epsilon_{i *}^{3}\right)$ for the models that all of the slow-roll parameters are small enough at the horizon crossing time. We stress that we evaluate the asymptotic values of spectral indices, say, the values at the end of inflation, by using the slow roll parameters evaluated at the horizon crossing time. This is the "horizon crossing formalism" itself.

To derive the runnings of spectral indices defined as

$$
\alpha_{I}:=\frac{d n_{I}}{d \ln k},
$$

we choose $k=k_{*}$, so that we can relate the spatial momentum to the horizon crossing time through $k=k_{*}=a_{*} H_{*}\left(n_{*}\right)$. By this, we get a relation between differentiations

\footnotetext{
${ }^{1}$ For other specific cases, see Appendix B.
} 


$$
\frac{d}{d \ln k}=\frac{d}{d \ln \left(a_{*} H_{*}\right)}=\frac{1}{1-\epsilon_{1 *}} \frac{d}{d n_{*}}
$$

Then, we can obtain the asymptotic values of the runnings of spectral indices up to the third order

$$
\begin{aligned}
\left.\alpha_{I}\left(k_{*}\right)\right|_{\left|k \tau_{I}\right| \rightarrow 0}= & -2 \epsilon_{1 *} \epsilon_{2 *}-\frac{1}{2}\left(3 f_{I 1 *} f_{I 2 *}-g_{I 1 *} g_{I 2 *}\right)-6 \epsilon_{1 *}^{2} \epsilon_{2 *}-2 \epsilon_{1 *}\left(2 f_{I 1 *} f_{I 2 *}-g_{I 1 *} g_{I 2 *}\right)-\frac{\epsilon_{1 *} \epsilon_{2 *}}{2}\left(5 f_{I 1 *}-3 g_{I 1 *}\right) \\
& -(2 C+2)\left(\epsilon_{1 *} \epsilon_{2 *}^{2}+\epsilon_{1 *} \epsilon_{2 *} \epsilon_{3 *}\right)-\frac{3}{2} f_{I 1 *}^{2} f_{I 2 *}+f_{I 1 *} f_{I 2 *} g_{I 1 *}+f_{I 1 *} g_{I 1 *} g_{I 2 *}-\frac{1}{2} g_{I 1 *}^{2} g_{I 2 *} \\
& -\frac{3 C+2}{2}\left(f_{I 1 *} f_{I 2 *}^{2}+f_{I 1 *} f_{I 2 *} f_{I 3 *}\right)+\frac{C+2}{2}\left(g_{I 1 *} g_{I 2 *}^{2}+g_{I 1 *} g_{I 2 *} g_{I 3 *}\right)+O\left(\epsilon_{i *}^{4}\right)
\end{aligned}
$$

where all of the slow roll parameters are evaluated at the horizon crossing time for the mode $k=k_{*}$.

As the simplest example, we consider the following case

$$
\begin{aligned}
\mathcal{F}_{s} & =\mathcal{G}_{s}=\epsilon_{1}, \\
\mathcal{F}_{t} & =\mathcal{G}_{t}=1,
\end{aligned}
$$

which is obtained for the canonical scalar field models with the potential. Using Eq. (57) and (60) for this case, up to the second order, we obtain

$$
\begin{aligned}
\left.n_{s}\left(k_{*}\right)\right|_{\left|k \tau_{s}\right| \rightarrow 0}= & 1-2 \epsilon_{1 *}-\epsilon_{2 *}-2 \epsilon_{1 *}^{2} \\
& -(2 C+3) \epsilon_{1 *} \epsilon_{2 *}-C \epsilon_{2 *} \epsilon_{3 *}, \\
\left.n_{t}\left(k_{*}\right)\right|_{\left|k \tau_{s}\right| \rightarrow 0}= & -2 \epsilon_{1 *}-2 \epsilon_{1 *}^{2}-2(C+1) \epsilon_{1 *} \epsilon_{2 *}, \\
\left.\alpha_{s}\left(k_{*}\right)\right|_{\left|k \tau_{s}\right| \rightarrow 0}= & -2 \epsilon_{1 *} \epsilon_{2_{*}}-\epsilon_{2 *} \epsilon_{3 *} \\
\left.\alpha_{t}\left(k_{*}\right)\right|_{\left|k \tau_{s}\right| \rightarrow 0}= & -2 \epsilon_{1 *} \epsilon_{2 *} .
\end{aligned}
$$

These correspond to the previous results $[23,26]$. In our formalism, however, the results are more rigorous and generic. While the previous results rely on an ansatz for $z_{I}$ or the Wentzel-Kramers-Brillouin approximation for the mode function, our slow roll expansion does never need any ansatzes nor approximations. Also, we can easily derive the spectral indices and their runnings for the generic models without deriving the power spectra themselves. Further, in [26], they perform an uncertain Taylor expansion for the asymptotic values of spectral indices to evaluate them by the slow roll parameters at the horizon crossing time. We showed explicitly, however, that we do not need such a Taylor expansion and can evaluate the spectral indices just by applying the horizon crossing formalism. We derived the runnings of spectral indices up to the third order also.

We comment on tiny effects coming from around the end of inflation. In typical scenarios, the inflation ends when the slow roll parameters rapidly grow and reach to $O(1)$ values. Thus, around the end, the slow roll expansion would break down. However, this hardly affects the final results since in the case the perturbations freeze out on superhorizon scales, the mode function remains almost the same as the asymptotic value (53). On the other hand, the exact ending time of the inflation differs from $\left|k \tau_{I}\right|=0$, and thus the asymptotic values of spectral indices have errors, which are represented by the $O\left(k \tau_{I}\right)$ term in Eq. (52). Those errors are, however, extremely small since at the end of inflation, $\left|k \tau_{I}\right| \sim \mathrm{e}^{-50}$ for the observational window of the mode $k$. Therefore, in substance, we can use the asymptotic values of the spectral indices (57) and their runnings (60) all the way to the end of the inflation.

We cannot apply, however, the slow roll expansion to the models in which the slow roll parameters grow above a certain limit nearby the horizon crossing time first, and then turn to small values again on the superhorizon scales. In these models, the mode function around the horizon crossing time behaves in a highly nontrivial way, and the contributions from $\Theta_{I}$ and/or $\tau_{I}$ deviate from the simple slow roll expansion significantly. We can further appreciate this by looking at the other expressions for the index (44). We study their behaviors in the next section.

\section{NUMERICAL ANALYSIS}

In this section, we perform a numerical analysis for the Starobinsky model [2]. First, we give a numerical setup for the background and the perturbation of the model. We focus on the scalar perturbation only since at the present, the tensor spectral index is not yet observed. Next, we compare the numerical result of slow roll expansion with the large $N_{*}$ expansion for the model. Then, we analyze the behavior of integration in Eq. (44), quoting the perturbation on the exact de Sitter background as a schematic example. We compare the numerical result of Eq. (44) for the scalar perturbation with the results of slow roll expansion and the large $N_{*}$ expansion also.

\section{A. Equations}

We consider the Starobinsky model which has the following potential

$$
V(\phi)=\frac{3}{4} M^{4}\left(1-\mathrm{e}^{-\sqrt{\frac{2}{3} \phi}}\right)^{2}
$$


in the Einstein frame. $M$ is a model parameter which has the mass dimension 1. The Friedmann equations and the equation of motion of the scalar field are expressed as

$$
\begin{aligned}
3 H^{2} & =\frac{1}{2} \dot{\phi}^{2}+V(\phi), \\
\dot{H} & =-\frac{\dot{\phi}}{2}, \\
\ddot{\phi}+3 H \dot{\phi}+V_{, \phi} & =0 .
\end{aligned}
$$

If we use the e-folding number (46) as a clock, we can rewrite Eqs. (64) as

$$
\begin{gathered}
H^{2}=\frac{V}{3-\phi_{, n}^{2} / 2}, \\
\frac{H_{, n}}{H}=-\frac{1}{2} \phi_{, n}^{2}, \\
\phi_{, n n}+\left(3+\frac{H_{, n}}{H}\right) \phi_{, n}+\frac{V_{, \phi}}{H^{2}}=0 .
\end{gathered}
$$

Combining Eqs. (65)-(67) gives the following single variable equation:

$$
\phi_{, n n}+\left(\phi_{, n}+\frac{V_{, \phi}}{V}\right)\left(3-\frac{1}{2} \phi_{, n}^{2}\right)=0 .
$$

We will use Eq. (68) as a background equation. The behavior of the Hubble parameter will be obtained by substituting the solution of Eq. (68) into Eq. (65). The relevant slow roll parameters for this model are given as

$$
\begin{gathered}
\epsilon_{1}=\frac{1}{2} \phi_{, n}^{2}, \\
\epsilon_{2}=-2\left(3-\frac{1}{2} \phi_{, n}^{2}\right)\left(1+\frac{V_{, \phi}}{\phi_{, n} V}\right), \\
\epsilon_{3}=\phi_{, n}^{2}+\frac{\phi_{, n}}{2} \frac{V_{, \phi}}{V}+\frac{3 V_{, \phi}}{\phi_{, n} V}+\frac{\frac{V_{, \phi \phi}}{V}-\frac{V_{, \phi \phi}^{2}}{V^{2}}}{1+\frac{V_{, \phi}}{\phi_{, n} V}} .
\end{gathered}
$$

While, the dynamics of the scalar perturbation $\psi_{s k}=u_{s k} / z_{s}$ is governed by the following equation:

$$
\psi_{s k, n n}+\left(3+\epsilon_{2}-\epsilon_{1}\right) \psi_{s k, n}+\frac{k^{2}}{a^{2} H^{2}} \psi_{s k}=0,
$$

which can be reduced from Eq. (23). Here, $z_{s}$ for the model was given by $z_{s}=a \sqrt{2 \epsilon_{1}}$.

Then, we define the end of inflation as the time when

$$
\epsilon_{1}\left(n_{\text {end }}\right)=1
$$

and we consider the scalar perturbation $\psi_{s k}$ which leave the horizon 54 e-folds before the end of inflation. Namely, we determine the wavenumber $k$ of perturbation as $k_{*}=$ $a_{*} H_{*}\left(n_{*}\right)$, where $n_{*}$ is satisfied with

$$
N_{*}:=\ln \left(\frac{a_{\text {end }}}{a_{*}}\right)=n_{\text {end }}-n_{*}=54 .
$$

Initial conditions We give the initial conditions and the mass parameter $M$ for the background as

$$
\begin{aligned}
\phi_{\text {in }} & =6.246 M_{\mathrm{pl}}, \\
\phi_{, n \text { in }} & =-10^{-2} M_{\mathrm{pl}}, \\
M & =1.419 \times 10^{-4} M_{\mathrm{pl}},
\end{aligned}
$$

so that we get

$$
\begin{aligned}
& H_{\text {in }} \simeq 10^{-8} M_{\mathrm{pl}} \sim \frac{E_{\mathrm{GUT}}^{2}}{M_{\mathrm{pl}}}, \\
& \epsilon_{\text {in }}=5.0 \times 10^{-5}, \\
& \epsilon_{\text {in }} \simeq 10^{-2} .
\end{aligned}
$$

The above initial conditions yield large e-folding number of the slow roll regime; $n_{\text {end }} \simeq 119.897$. The background approaches rapidly to the slow roll attractor regime where $\dot{\phi} \simeq-V_{, \phi} / 3 H$, so that after a few e-folds, the dynamics of $\phi(t)$ is hardly affected by the choice of initial values.

For the scalar perturbation, because of the problem of calculation costs, we will take $n_{\text {pin }}$ as an initial e-folding number which satisfies $n_{\text {end }}-n_{\text {pin }}=64$. Then, we will redefine $n$ as $n-n_{\text {pin }}$, so that in the new definition, we obtain $n_{\text {pin }}=0, n_{*}=10$, and $n_{\text {in }} \simeq-55.897$. At the time $n_{\text {pin }}=0$, the perturbation which leave the horizon at $n_{*}=$ 10 stays deep inside the horizon yet, so we approximate its initial values by those on the exact de Sitter background. Using Eq. (51) with $\tau_{s} \simeq-1 / a H$, we give the initial values of scalar perturbation at $n_{\text {pin }}$ as

$$
\begin{aligned}
\psi_{s k}\left(n_{\mathrm{pin}}\right) & \left.\simeq \frac{u_{s k}^{(0)}}{z_{s}}\right|_{\mathrm{pin}}=\left[\frac{1}{z_{s} \sqrt{2 k_{*}}}+i \frac{a H}{z_{s} \sqrt{2 k_{*}^{3}}}\right]_{\mathrm{pin}} \\
& =:\left[\pi_{s k}+i \sigma_{s k}\right]_{\mathrm{pin}},
\end{aligned}
$$

$$
\begin{aligned}
\psi_{s k, n}\left(n_{\mathrm{pin}}\right) \simeq & {\left[\pi_{s k}\left(-\frac{z_{s, n}}{z_{s}}+\frac{1}{1+k_{*}^{2} /\left(a^{2} H^{2}\right)}\right)\right.} \\
& \left.+\sigma_{s k} \frac{k_{*}^{3} /\left(a^{3} H^{3}\right)}{1+k_{*}^{2} /\left(a^{2} H^{2}\right)}\right]\left.\right|_{\mathrm{pin}} \\
& +i\left\{-\pi_{s k} \frac{k_{*}^{3} /\left(a^{3} H^{3}\right)}{1+k_{*}^{2} /\left(a^{2} H^{2}\right)}\right. \\
& \left.+\sigma_{s k}\left[-\frac{z_{s, n}}{z_{s}}+\frac{1}{1+k_{*}^{2} /\left(a^{2} H^{2}\right)}\right]\right\}_{\mathrm{pin}},
\end{aligned}
$$


where $\pi_{s k}$ and $\sigma_{s k}$ are the real and the imaginary part of $\psi_{s k}$, respectively. We set the initial phase of the mode function $u_{s k}^{(0)}$ to be zero for simplicity, which can be absorbed into the initial phase of the vacuum.

\section{B. Slow roll expansion vs the large $N_{*}$ expansion}

Using the slow roll expansion, the spectral index $n_{s}$ for the Starobinsky model is derived as Eq. (62), and its numerical value gives

$$
\begin{aligned}
n_{s}\left(k_{*}\right) & \simeq\left[1-2 \epsilon_{1}-\epsilon_{2}-2 \epsilon_{1}^{2}-(2 C+3) \epsilon_{1} \epsilon_{2}-C \epsilon_{2} \epsilon_{3}\right]_{n=n_{*}} \\
& \simeq 0.9643 .
\end{aligned}
$$

Whereas, the large $N_{*}$ expansion up to $O\left(1 / N_{*}^{2}\right)$ for Eq. (62), in which the slow roll parameters are expanded by $1 / N_{*}$, gives the following expression [43]:

$$
\begin{aligned}
n_{s}\left(k_{*}\right) & \simeq 1-\frac{2}{N_{*}}+\left(0.81+\frac{3}{2} \ln \left(N_{*}\right)\right) \frac{1}{N_{*}^{2}} \\
& \simeq 0.9653 .
\end{aligned}
$$

These two expansions differ slightly within the range of 1-2\%o. Since the large $N_{*}$ expansion here is just an approximation up to $O\left(1 / N_{*}^{2}\right)$ and the definition of $N_{*}$ is entirely based on the slow roll approximation, ${ }^{2}$ the result based on the slow roll expansion (79) would be more reliable than that of the large $N_{*}$ expansion (80). If the sensitivity of future observations can be up to $O\left(10^{-3}\right)$ for the scalar spectral index, it would be better to use numerical results of the slow roll expansion as the theoretical prediction for a fixed $N_{*}$.

\section{Analysis of the other expression for the spectral index}

Here, we use the expression (44) for the scalar perturbation

\footnotetext{
${ }^{2}$ In the large $N_{*}$ expansion, the end of inflation is defined as the time when

$$
\epsilon_{v}:=\frac{M_{\mathrm{pl}}^{2}}{2}\left(\frac{V_{, \phi}}{V}\right)^{2}=1 .
$$

While, the e-folding number from the end to the horizon crossing time is defined as

$$
N_{*}^{\text {large }}:=\frac{1}{M_{\mathrm{pl}}^{2}} \int_{\phi_{\mathrm{end}}}^{\phi_{*}} \frac{V}{V_{\tilde{\phi}}} d \tilde{\phi},
$$

which does not include the $\dot{\phi}$ dependence of $N_{*}$. In the large $N_{*}$ expansion, we expand the slow roll parameters defined in Eq. (47) by the above $N_{*}^{\text {large }}$ and calculate physical quantities like the spectral index. See [43] for the details.
}

$n_{s}=2-4 \operatorname{Im}\left[\frac{\psi_{s k}^{* 2}\left(\tau_{s}\right)}{\left|\psi_{s k}\left(\tau_{s}\right)\right|^{2}} \int_{-\infty(1+i \varepsilon)}^{\tau_{s}} z_{s}^{2}\left(\partial_{\tilde{\tau}_{s}} \psi_{s k}\right)^{2} d \tilde{\tau}_{s}\right]$

to evaluate the scalar spectral index $n_{s}$.

First, as a schematic example, we consider the above expression for the perturbation on the exact de Sitter background. In that case, we obtain the exact solution for the mode function as Eq. (51), and we give $z_{s}=a$. Using a new variable $x_{s}:=k \tau_{s}$ and Eq. (45), the expression (81) reduces to

$$
\begin{aligned}
n_{s}^{\mathrm{dS}}= & 2-4 \operatorname{Im}\left[-\frac{1-x_{s}^{2}-2 i x_{s}}{1+x_{s}^{2}} \mathrm{e}^{2 i x_{s}}\right. \\
& \left.\times \int_{-\infty(1+i \varepsilon)}^{x_{s}}-\frac{1}{2} \mathrm{e}^{-2 i \tilde{x}_{s}} d \tilde{x}_{s}\right] .
\end{aligned}
$$

Note that $x_{s}$ is always a negative number. We can easily perform the integration and get the exact solution

$$
n_{s}^{\mathrm{dS}}=1+\frac{2 x_{s}^{2}}{1+x_{s}^{2}}
$$

which is consistent with a direct calculation from Eq. (51), (37), and (38). Then, we consider Eq. (82) for the time $\left|x_{\text {send }}\right| \ll 1$. Up to the second order of $x_{\text {send }}$, we obtain

$$
\begin{aligned}
\left.n_{s}^{\mathrm{dS}}\right|_{\mathrm{end}} & \simeq 2-4 \operatorname{Im}\left[-1 \cdot-\frac{1}{2} \int_{-\infty(1+i \varepsilon)}^{x_{\text {send }}} \mathrm{e}^{-2 i \tilde{x}_{s}} d \tilde{x}_{s}\right] \\
& =2-\cos 2 x_{s \text { end }} \\
& \simeq 1+2 x_{s \text { end }}^{2} .
\end{aligned}
$$

This is also consistent to the exact solution (83) up to the second order. We find that only the imaginary part of the above integration contributes to the spectral index at the end $\left|x_{\text {send }}\right| \ll 1$. For a further analysis, we separate the integration in (84) as follows:

$$
\begin{aligned}
\operatorname{Im} & {\left[\int_{-\infty(1+i \varepsilon)}^{x_{\text {send }}} \mathrm{e}^{-2 i \tilde{x}_{s}} d \tilde{x}_{s}\right] } \\
& =\operatorname{Im}\left[\left(\int_{x_{s>}}^{x_{\text {send }}}+\int_{x_{s \Delta}}^{x_{s>}}+\int_{-\infty(1+i \varepsilon)}^{x_{s}}\right) \mathrm{e}^{-2 i \tilde{x}_{s}} d \tilde{x}_{s}\right],
\end{aligned}
$$

where $x_{s>}$ and $x_{s \triangle}$ satisfy

$$
1 \gg\left|x_{s>}\right| \gg\left|x_{\text {send }}\right|, \quad \sin 2 x_{s \triangle}= \pm 1
$$

$x_{s \triangle}$ is the time when $\sin 2 x_{s}$ is at any of extrema, and thus the third part of integration becomes zero: 


$$
\begin{aligned}
\operatorname{Im} & {\left[\int_{-\infty(1+i \varepsilon)}^{x_{s \Delta}} \mathrm{e}^{-2 i \tilde{x}_{s}} d \tilde{x}_{s}\right] } \\
& =\operatorname{Im}\left[\frac{1}{2}\left(\sin 2 \tilde{x}_{s}+i \cos 2 \tilde{x}_{s}\right)\right]_{-\infty(1+i \varepsilon)}^{x_{s \Delta}}=0
\end{aligned}
$$

This implies roughly that from the subhorizon scales, there is no contribution to the spectral index at the time $x_{\text {send }}$ since the integrand oscillates periodically. We take $x_{s \Delta}$ as the time corresponding to the last extremum of the sine function after the horizon crossing. The first part of integration (85) is the contribution to $n_{s}^{\mathrm{dS}}\left(x_{\text {send }}\right)$ from the superhorizon scales where $\left|x_{s}\right| \ll 1$, and the second part is that from only a few e-folds around the horizon crossing. Up to the second order of $x_{s>}$, the contribution from the first part becomes

$$
\begin{aligned}
\operatorname{Im}\left[\int_{x_{s>}}^{x_{\text {send }}} \mathrm{e}^{-2 i \tilde{x}_{s}} d \tilde{x}_{s}\right] & =\operatorname{Im}\left[\int_{x_{s>}}^{x_{\text {send }}}-i \sin 2 \tilde{x}_{s} d \tilde{x}_{s}\right] \\
& =\frac{1}{2}\left[\cos 2 x_{\text {send }}-\cos 2 x_{s>}\right] \\
& \simeq x_{s>}^{2},
\end{aligned}
$$

where we ignore $O\left(x_{\text {send }}^{2}\right)$ terms. This shows clearly that there is less contributions to $n_{s}^{\mathrm{dS}}\left(x_{\text {send }}\right)$ from the superhorizon scales, which matches to the conservation of the perturbation (see Fig. 1). In the following, we ignore the contribution from the superhorizon scales. Eventually, in Eq. (84), we can reduce the integration region to around the

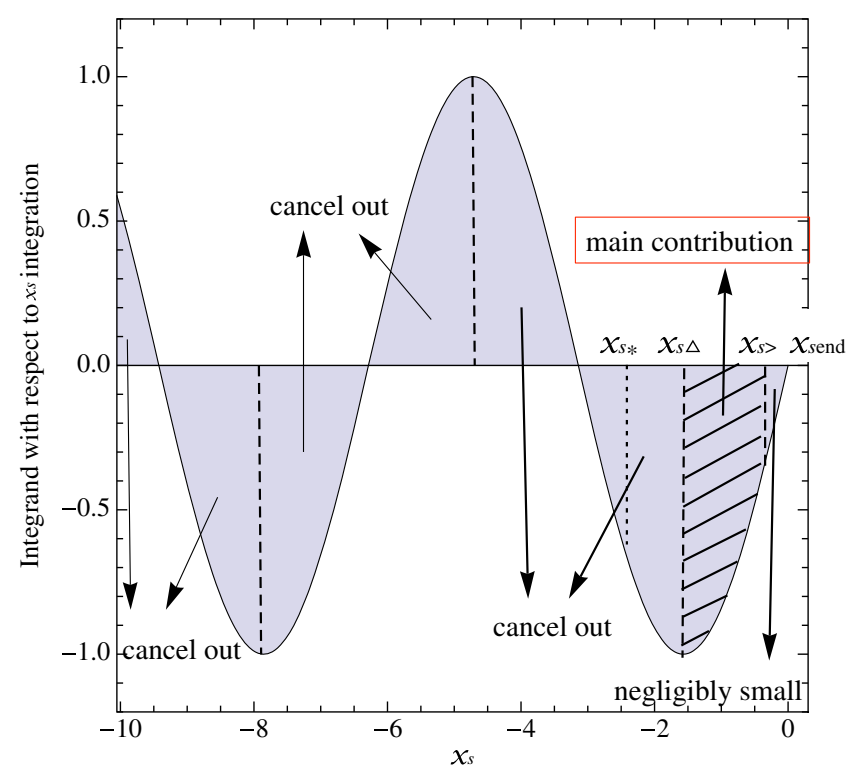

FIG. 1. Schematic picture of the integration in Eq. (84). Main contribution of the integration comes from the last quarter period of the imaginary part of integrand since the integrand with respect to $x_{s}$ is expressed by the sine function. horizon crossing time only and extract the imaginary part of the integrand:

$$
\begin{aligned}
\left.n_{s}^{\mathrm{dS}}\right|_{\mathrm{end}} & \simeq 2-4 \operatorname{Im}\left[-1 \cdot \frac{i}{2} \int_{x_{s \Delta}}^{x_{s>}} \sin 2 \tilde{x}_{s} d \tilde{x}_{s}\right] \\
& =1+O\left(x_{s>}^{2}\right) .
\end{aligned}
$$

The accuracy of integration is up to $O\left(x_{s>}^{2}\right)$.

We expect a similar behavior of integrand for the generic case also. The situation, however, changes importantly because the slow roll parameters will deform the integrand from the exact de Sitter case. We can understand the effect of slow roll parameters by looking at the MS equation (23) for the generic cases

$$
\begin{aligned}
\partial_{\tau_{s}}^{2} u_{s k} & +\left[k^{2}+m_{s}^{2}\left(\tau_{s}\right)\right] u_{s k}=0, \\
m_{s}^{2}= & -\frac{\partial_{\tau_{s}}^{2} z_{s}}{z_{s}} \\
= & -\frac{a^{2} H^{2}}{c_{s}^{2}}\left\{2-\epsilon_{1}+\frac{1}{4}\left(f_{s 1}+5 g_{s 1}\right)\right. \\
& -\frac{1}{4}\left[\left(f_{s 1}+g_{s 1}\right)\left(\epsilon_{1}+\frac{1}{4}\left(f_{s 1}-3 g_{s 1}\right)\right)\right. \\
& \left.\left.-f_{s 1} f_{s 2}-g_{s 1} g_{s 2}\right]\right\} .
\end{aligned}
$$

In the subhorizon scales where $c_{s} k \gg a H$, the oscillation term dominates over the mass term if the slow roll parameters remain not significantly big. In such a region, the mode function does not feel its mass during one period. Thus, as in Fig. 1, the cancellation by the oscillation takes place in the deep subhorizon scales even if the slow roll parameters deform the mass. While, in the superhorizon scales where $c_{s} k \ll a H$, the mass term dominates over the oscillation term and the mode function $u_{s k}$ rapidly approaches to the asymptotic value (28). Even if the slow roll expansion breaks down at the region $c_{s} k \ll a H$, the asymptotic behavior of mode function pursues as long as the mass term dominates enough over the oscillation term. Thus, there is less contribution from the superhorizon scales also even for the generic cases. The breakdown of slow roll expansion on the superhorizon scales implies a deformation of just the tiny fraction between $x_{s>}$ and $x_{\text {send }}$ in Fig. 1, which remains still negligible. On the other hand, the mode function can be significantly deformed around the horizon crossing time, since it can feel the deformation of mass from the exact de Sitter case during a few e-folds around the horizon crossing time. Therefore, unlike the de Sitter case, we need to choose $x_{s \triangle}$ as the time when the imaginary part of integrand (81) is at the extremum a few e-folds before the horizon crossing time for the generic cases.

Following the speculation in the above, we reduce the integration region of Eq. (81) in terms of $n$ as 
$\left.n_{s}\right|_{\text {end }} \simeq 2-4 \operatorname{Im}\left[\frac{\psi_{s k}^{* 2}\left(n_{\text {end }}\right)}{\left|\psi_{s k}\left(n_{\text {end }}\right)\right|^{2}} \int_{n_{\Delta}}^{n_{>}} z_{s}^{2} a H \psi_{s k, \tilde{n}}^{2} d \tilde{n}\right]$,

where $n_{>}$is a time a few e-folds after the horizon crossing and $n_{\Delta}$ is a time when the imaginary part of integrand is at the extremum. With use of the decomposition $\psi_{s k}=$ $\pi_{s k}+i \sigma_{s k}$, we write the prefactor of integration as

$$
\left.\frac{\psi_{s k}^{* 2}}{\left|\psi_{s k}\right|^{2}}\right|_{\text {end }}=\left.\frac{\pi_{s k}^{2}-\sigma_{s k}^{2}-2 i \pi_{s k} \sigma_{s k}}{\pi_{s k}^{2}+\sigma_{s k}^{2}}\right|_{\text {end }} .
$$

In the de Sitter example, we can safely ignore the imaginary part of the prefactor since $\pi_{s k}$ for the de Sitter case approaches to zero on the superhorizon scales. However, it is not for the present case because we changed the argument between $\pi_{s k}$ and $\sigma_{s k}$ to perform the numerical calculation. Thus, to apply the analysis obtained from the de Sitter case for the present case, we need to tune the phase of $\psi_{s k}$ so that the real part of $\psi_{s k}\left(n_{\text {end }}\right)$ vanishes. We can always perform this procedure without a loss of generality by adding the phase factor to $\psi_{s k}$ as

$$
\begin{aligned}
\psi_{s k}^{\vartheta} & =\pi_{s k}^{\vartheta}+i \sigma_{s k}^{\vartheta}:=\psi_{s k} \mathrm{e}^{i \vartheta}, \\
\cos \vartheta & =\left.\frac{\sigma_{s k}}{\sqrt{\pi_{s k}^{2}+\sigma_{s k}^{2}}}\right|_{\text {end }}, \\
\sin \vartheta & =\left.\frac{\pi_{s k}}{\sqrt{\pi_{s k}^{2}+\sigma_{s k}^{2}}}\right|_{\text {end }},
\end{aligned}
$$

which leads to $\pi_{s k}^{\vartheta}\left(n_{\text {end }}\right)=0$. Then, using $\psi_{s k}^{\vartheta}$, we can rewrite Eq. (91) as

$\left.n_{s}\right|_{\mathrm{end}} \simeq 2-4 \operatorname{Im}\left[-1 \cdot i \int_{n_{\Delta}}^{n_{>}} 2 z_{s}^{2} a H\left(\pi_{s k, \tilde{n}}^{\vartheta} \sigma_{s k, \tilde{n}}^{\vartheta}\right) d \tilde{n}\right]$,

where

$$
\begin{aligned}
\pi_{s k, n}^{\vartheta} \sigma_{s k, n}^{\vartheta}= & \left(\pi_{s k, n}^{2}-\sigma_{s k, n}^{2}\right) \cos \vartheta \sin \vartheta \\
& +\pi_{s k, n} \sigma_{s k, n}\left(\cos ^{2} \vartheta-\sin ^{2} \vartheta\right) .
\end{aligned}
$$

The actual behaviors of the integrand in Eq. (94) are shown in Figs. 2, 3.

As shown in Fig. 3, the last extremum of the integrand in terms of $n$ is at $n=10.22055$, which is the e-folds soon after the horizon crossing time $n_{*}=10$. Then, we choose $n_{\Delta}$ as the time at the extremum before $n_{\triangle}=10.22055$ and evaluate Eq. (94) by choosing enough large value for $n_{>}$. The results are shown in Table I. It shows that the deformation of the integrand from the exact de Sitter case causes the red spectral tilt, and $\left.n_{s}\right|_{\text {end }}$ seems to converge into the value 0.9646 . This result is consistent with both of the slow roll expansion method and the large $N_{*}$ expansion method within the range of $1 \%$ differences. The slight

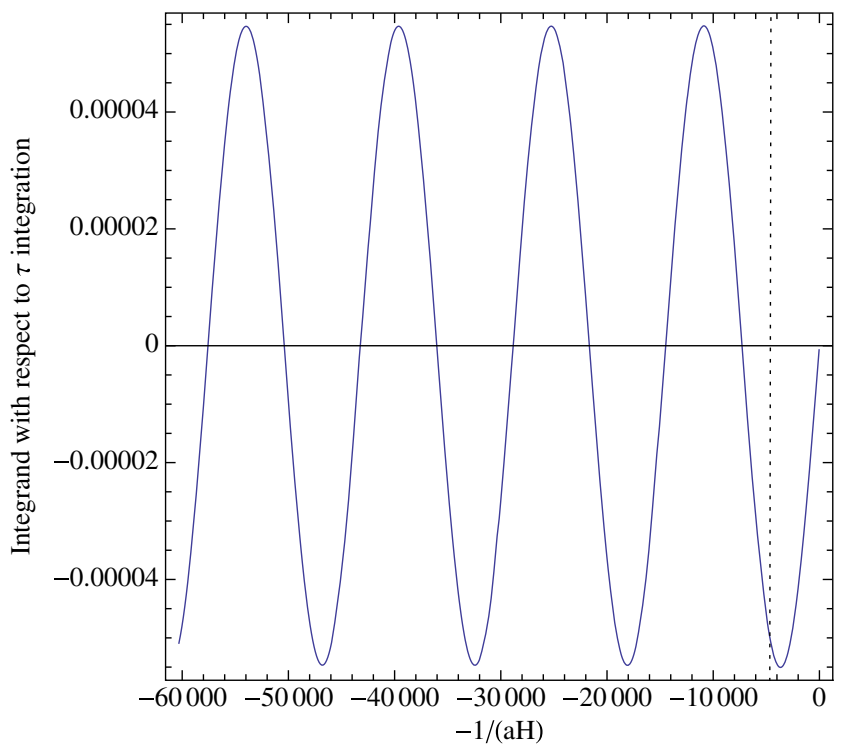

FIG. 2. The integrand in Eq. (94) with respect to $-1 /(a H) \simeq \tau_{s}$. We multiplied $a H$ to the integrand since here, we change the measure as $d n=a H d \tau_{s}$. Similarly to the de Sitter case, the integrand (multiplied $a H$ ) almost behaves as the sine function.

difference would originate from the approximations for the integration region and for the initial values of the mode function (77).

As we expected, the numerical value of spectral index from Eq. (94) almost corresponds to that of horizon crossing formalism based on the slow roll expansion. Thus, the analysis we performed for the integration region is legitimate. To calculate the spectral index, it is the most

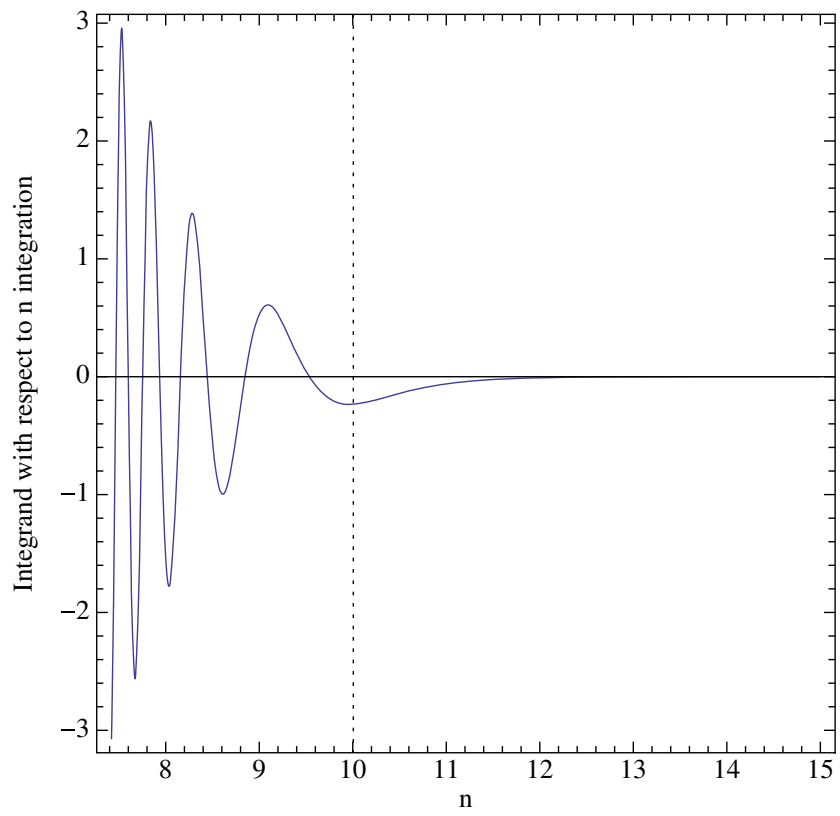

FIG. 3. The integrand in Eq. (94) with respect to $n$. The last extremum appears after the horizon crossing time $n_{*}=10$. 
TABLE I. Values of $\left.n_{s}\right|_{\text {end }}$ with respect to the position of $n_{\triangle}$.

\begin{tabular}{lccc}
\hline \hline$i$-th last extremum & $n_{\Delta}$ & $n_{>}$ & $\left.n_{s}\right|_{\text {end }}$ \\
\hline 1 & 10.22055 & 15 & 0.9759 \\
2 & 9.13269 & 15 & 0.9632 \\
3 & 8.62547 & 15 & 0.9648 \\
4 & 8.29068 & 15 & 0.9644 \\
5 & 8.04033 & 15 & 0.9646 \\
6 & 7.84027 & 15 & 0.9645 \\
7 & 7.67365 & 15 & 0.9646 \\
8 & 7.53086 & 15 & 0.9646 \\
\hline \hline
\end{tabular}

important to know the behaviors of the mode function and the slow roll parameters around the horizon crossing time. In turn, even if the slow roll expansion breaks down around the horizon crossing time only, we can no longer apply the horizon crossing formalism to the spectral index. This is because the mass around the crossing will be strongly deformed from the de Sitter case. We note that even if all the slow roll parameters are smaller than $O(1)$ values, the slow roll expansion can break when we cannot use the slow roll parameters as the expansion parameters. Hence, if the potential has a bump and/or a cliff and the slow roll expansion breaks there, we need to perform the numerical analysis for the spectral index as here instead of using the horizon crossing formalism. We can apply the same analysis to the tensor perturbation also.

\section{SUMMARY}

In this article, we have directly derived the two exact expressions of the spectral indices from the WardTakahashi identity associated with the dilatation charge. We use generic free actions for the scalar and the tensor perturbations, so that we can apply the expressions to all of the single field inflation models which possess the Lorentzinvariant scaling. In the slow roll expansion, we have used one of the expressions (42) and have derived the spectral indices up to the second order of the slow roll parameters. Applying our expression to the canonical scalar model with the potential, the result is consistent with the previous works. Our formalism is, however, more rigorous and generic since we do not need any ansatzes nor approximations to the models and is therefore applicable to much more models than before. We have revealed that in the slow roll expansion, to evaluate the final values of the spectral indices at the end of inflation, we just need the horizon crossing formalism in which we change the time in the final values to the time when $a_{*} H_{*}=k_{*}$, since the final values do not depend on the time actually. We have also derived the runnings of the spectral indices up to the third order.

To understand how the perturbations and the background contribute to the spectral indices, we have analyzed the other expression of the spectral indices (44). First, we analyzed the scalar perturbation on the exact de Sitter background as a schematic example. Then, we applied the same method to the Starobinsky model and performed the numerical calculation. We found that the contributions to the spectral indices are only from around the horizon crossing time, and there is less contribution from the sub and the superhorizon scales. The spectral tilts are intrinsically connected to the masses of perturbations, deformed by the slow roll parameters, around the horizon crossing time. This is because only around that time, the perturbations can feel the deformation of masses from the exact de Sitter case. In turn, this result indicates that even if the slow roll expansion breaks down around the horizon crossing time only, we can no longer apply the slow roll expansion since the mode functions around the horizon crossing time significantly deviate from those of the slow roll expansion. In such a case, the approximate expression (94) we have derived will be a powerful tool to evaluate the spectral indices.

In Sec. IV, we have evaluated the values of the scalar spectral index in several methods. It was shown that there is $1 \%$ o discrepancy between horizon crossing formalism and the large $N_{*}$ expansion in the Starobinsky model. In fact, the large $N_{*}$ expansion only has $1 \%$ o level precision, therefore, this is an expected result. On the other hand, our method is consistent with both the horizon crossing formalism and the large $N_{*}$ expansion in 1\%o accuracy. Therefore, our method may be regarded as a useful indicator of $n_{s}$, similar to that using the large $N_{*}$ expansion.

\section{ACKNOWLEDGMENTS}

The authors thank Toshiya Namikawa and Misao Sasaki for fruitful discussions. The authors' work is partially supported by Leung Center for Cosmology and Particle Astrophysics, National Taiwan Univ. (FI121).

\section{APPENDIX A: DERIVATION OF ANOTHER FORM OF THE SPECTRAL INDEX}

First, we deform the integration included in $\Theta_{I}$ (43) as follows;

$$
\begin{aligned}
\frac{u_{I k}^{* 2}}{\left|u_{I k}\right|^{2}} \int_{-\infty}^{\tau_{I}} \theta_{I} u_{I k}^{2} d \tilde{\tau}_{I} \\
=\frac{u_{I k}^{* 2}}{\left|u_{I k}\right|^{2}} \int_{-\infty}^{\tau_{I}}\left(\frac{m_{I}^{2}}{2} u_{I k}^{2}-\tilde{\tau}_{I} \partial_{\tilde{\tau}_{I}} u_{I k} \cdot m_{I}^{2} u_{I k}\right) d \tilde{\tau}_{I} \\
\quad-\frac{u_{I k}^{* 2}}{\left|u_{I k}\right|^{2}} \frac{\tau_{I 0}}{2} m_{I 0}^{2} u_{I k 0}^{2}+\text { real part },
\end{aligned}
$$

where the quantities with subscript 0 are evaluated at $\tau_{I 0}=-\infty$. We do not show the real parts explicitly because they do not contribute to $\Theta_{I}$. The mass at the past infinity approaches to zero faster than $1 / \tau_{I}$ if we extrapolate the slow roll parameters as they remain finite at the past 
infinity, so the second term in the right-hand side becomes zero. Then, using the equation of motion for $u_{I k}$, we reduce Eq. (A1) to

$$
\begin{aligned}
\frac{u_{I k}^{* 2}}{\left|u_{I k}\right|^{2}} \int_{-\infty}^{\tau_{I}} \theta_{I} u_{I k}^{2} d \tilde{\tau}_{I} & =\frac{u_{I k}^{* 2}}{\left|u_{I k}\right|^{2}} \int_{-\infty}^{\tau_{I}}\left[\frac{m_{I}^{2}}{2} u_{I k}^{2}+\tilde{\tau}_{I} \partial_{\tilde{\tau}_{I}} u_{I k}\left(\partial_{\tilde{\tau}_{I}}^{2} u_{I k}+k^{2} u_{I k}\right)\right] d \tilde{\tau}_{I}+\text { real part } \\
& =\frac{u_{I k}^{* 2}}{\left|u_{I k}\right|^{2}} \int_{-\infty}^{\tau_{I}}\left[\frac{m_{I}^{2}}{2} u_{I k}^{2}-\frac{1}{2}\left(\partial_{\tilde{\tau}_{I}} u_{I k}\right)^{2}-\frac{k^{2}}{2} u_{I k}^{2}\right] d \tilde{\tau}_{I}+\frac{u_{I k}^{* 2}}{\left|u_{I k}\right|^{2}}\left[\frac{\tilde{\tau}_{I}}{2}\left(\left(\partial_{\tilde{\tau}_{I}} u_{I k}\right)^{2}+k^{2} u_{I k}^{2}\right)\right]_{-\infty}^{\tau_{I}}+\text { real part } \\
& =\frac{u_{I k}^{* 2}}{\left|u_{I k}\right|^{2}} \int_{-\infty}^{\tau_{I}}\left[\frac{m_{I}^{2}}{2} u_{I k}^{2}-\frac{1}{2}\left(\partial_{\tilde{\tau}_{I}} u_{I k}\right)^{2}-\frac{k^{2}}{2} u_{I k}^{2}\right] d \tilde{\tau}_{I}+\frac{\tau_{I}}{2} \frac{u_{I k}^{* 2}}{\left|u_{I k}\right|^{2}}\left(\partial_{\tau_{I}} u_{I k}\right)^{2}+\text { real part, }
\end{aligned}
$$

where we used Eq. (32) at the last equality. We can further deform this integration as

$$
\begin{aligned}
\frac{u_{I k}^{* 2}}{\left|u_{I k}\right|^{2}} \int_{-\infty}^{\tau_{I}} \theta_{I} u_{I k}^{2} d \tilde{\tau}_{I} & =\frac{u_{I k}^{* 2}}{\left|u_{I k}\right|^{2}} \int_{-\infty}^{\tau_{I}}\left[-\frac{1}{2}\left(\partial_{\tilde{\tau}_{I}}^{2} u_{I k}+k^{2} u_{I k}\right) u_{I k}-\frac{1}{2}\left(\partial_{\tilde{\tau}_{I}} u_{I k}\right)^{2}-\frac{k^{2}}{2} u_{I k}^{2}\right] d \tilde{\tau}_{I}+\frac{\tau_{I}}{2} \frac{u_{I k}^{* 2}}{\left|u_{I k}\right|^{2}}\left(\partial_{\tau_{I}} u_{I k}\right)^{2}+\text { real part } \\
& =-\frac{u_{I k}^{* 2}}{\left|u_{I k}\right|^{2}} \int_{-\infty}^{\tau_{I}} k^{2} u_{I k}^{2} d \tilde{\tau}_{I}-\frac{1}{2} u_{I k}^{*} \partial_{\tau_{I}} u_{I k}+\frac{1}{2} \frac{u_{I k}^{* 2}}{\left|u_{I k}\right|^{2}} u_{I k 0} \partial_{\tau_{I}} u_{I k 0}+\frac{\tau_{I}}{2} \frac{u_{I k}^{* 2}}{\left|u_{I k}\right|^{2}}\left(\partial_{\tau_{I}} u_{I k}\right)^{2}+\text { real part. }
\end{aligned}
$$

Eliminating the $k^{2}$ term from Eqs. (A2) and (A3), we obtain

$$
\frac{u_{I k}^{* 2}}{\left|u_{I k}\right|^{2}} \int_{-\infty}^{\tau_{I}} \theta_{I} u_{I k}^{2} d \tilde{\tau}_{I}=\frac{u_{I k}^{* 2}}{\left|u_{I k}\right|^{2}} \int_{-\infty}^{\tau_{I}}\left[m_{I}^{2} u_{I k}^{2}-\left(\partial_{\tilde{\tau}_{I}} u_{I k}\right)^{2}\right] d \tilde{\tau}_{I}+\frac{1}{2} u_{I k}^{*} \partial_{\tau_{I}} u_{I k}+\frac{\tau_{I}}{2} \frac{u_{I k}^{* 2}}{\left|u_{I k}\right|^{2}}\left(\partial_{\tau_{I}} u_{I k}\right)^{2}-\frac{1}{2} \frac{u_{I k}^{* 2}}{\left|u_{I k}\right|^{2}} u_{I k 0} \partial_{\tau_{I}} u_{I k 0}+\text { real part. }
$$

We take the following contour for the integration in Eq. (A4):

$$
\int_{-\infty}^{\tau_{I}}\left(m_{I}^{2} u_{I k}^{2}-\left(\partial_{\tilde{\tau}_{I}} u_{I k}\right)^{2}\right) d \tilde{\tau}_{I}=\left(\int_{-\infty(1+i \varepsilon)}^{\tau_{I}}+\int_{-\infty}^{-\infty(1+i \varepsilon)}\right)\left[m_{I}^{2} u_{I k}^{2}-\left(\partial_{\tilde{\tau}_{I}} u_{I k}\right)^{2}\right] d \tilde{\tau}_{I}
$$

where we apply the $i \varepsilon$ prescription so as to calculate the above integration easily. We are not aware of the implication of this prescription. However, we do not need to take care about it since the extrapolated mode function before the inflation does not need to follow the actual history of the universe. It is enough to set the contour at the past infinity calculable. We extrapolate the mode function at the second contour as the same form as Eq. (32). Then, the integration from the second contour gives

$$
\int_{-\infty}^{-\infty(1+i \varepsilon)}\left[m_{I}^{2} u_{I k}^{2}-\left(\partial_{\tilde{\tau}_{I}} u_{I k}\right)^{2}\right] d \tilde{\tau}_{I}=\int_{-\infty}^{-\infty(1+i \varepsilon)} \frac{k}{2} \mathrm{e}^{-2 i k \tilde{\tau}_{I}} d \tilde{\tau}_{I}=-\left.\frac{i}{4} \mathrm{e}^{-2 i k \tau_{I}}\right|_{\tau_{I}=-\infty}=\frac{1}{2} u_{I k 0} \partial_{\tau_{I}} u_{I k 0} .
$$

Then, Eq. (A4) reduces to

$$
\frac{u_{I k}^{* 2}}{\left|u_{I k}\right|^{2}} \int_{-\infty}^{\tau_{I}} \theta_{I} u_{I k}^{2} d \tilde{\tau}_{I}=\frac{u_{I k}^{* 2}}{\left|u_{I k}\right|^{2}} \int_{-\infty(1+i \varepsilon)}^{\tau_{I}}\left[m_{I}^{2} u_{I k}^{2}-\left(\partial_{\tilde{\tau}_{I}} u_{I k}\right)^{2}\right] d \tilde{\tau}_{I}+\frac{1}{2} u_{I k}^{*} \partial_{\tau_{I}} u_{I k}+\frac{\tau_{I}}{2} \frac{u_{I k}^{* 2}}{\left|u_{I k}\right|^{2}}\left(\partial_{\tau_{I}} u_{I k}\right)^{2}+\text { real part. }
$$

Substituting this to Eq. (43), we get

$$
\begin{aligned}
\Theta_{I} & =\operatorname{Im}\left[2 u_{I k}^{*} \partial_{\tau_{I}} u_{I k}+\frac{2 \tau_{I}}{\left|u_{I k}\right|^{2}} u_{I k}^{* 2}\left(\partial_{\tau_{I}} u_{I k}\right)^{2}+\frac{4 u_{I k}^{* 2}}{\left|u_{I k}\right|^{2}} \int_{-\infty(1+i \varepsilon)}^{\tau_{I}}\left(m_{I}^{2} u_{I k}^{2}-\left(\partial_{\tilde{\tau}_{I}} u_{I k}\right)^{2}\right) d \tilde{\tau}_{I}\right] \\
& =-1-2 \tau_{I} \frac{\partial_{\tau_{I}}\left|u_{I k}\right|}{\left|u_{I k}\right|}+4 \operatorname{Im}\left[\frac{u_{I k}^{* 2}}{\left|u_{I k}\right|^{2}} \int_{-\infty(1+i \varepsilon)}^{\tau_{I}}\left(m_{I}^{2} u_{I k}^{2}-\left(\partial_{\tilde{\tau}_{I}} u_{I k}\right)^{2}\right) d \tilde{\tau}_{I}\right]
\end{aligned}
$$


where we used the Wronskian (24) at the second equality. Introducing $\psi_{I k}=u_{I k} / z_{I}$ and performing a integration by parts, we obtain the spectral index in the other generic form (44)

$$
n_{I}=\mathcal{N}_{I}+1-4 \operatorname{Im}\left[\frac{\psi_{I k}^{* 2}}{\left|\psi_{I k}\right|^{2}} \int_{-\infty(1+i \varepsilon)}^{\tau_{I}} z_{I}^{2}\left(\partial_{\tilde{\tau}_{I}} \psi_{I k}\right)^{2} d \tilde{\tau}_{I}\right] .
$$

\section{APPENDIX B: SPECIFIC MODELS FOR THE SLOW ROLL EXPANSION}

\section{Power-law inflation}

We first consider the power-law inflation model as one of specific models. The scale factor and the coefficients in the free action are given by

$$
\begin{aligned}
a & \propto t^{1 / \epsilon_{1}} \\
\mathcal{F}_{s} & =\mathcal{G}_{s}=\epsilon_{1}=\text { constant }, \\
\mathcal{F}_{t} & =\mathcal{G}_{t}=1 .
\end{aligned}
$$

In this case, for the scalar and the tensor perturbations, the conformal times and the masses become the same ones

$$
\begin{gathered}
\tau_{s}=\tau_{t}=-\frac{1}{a H\left(1-\epsilon_{1}\right)}, \\
m_{I}^{2}=-\frac{\partial_{\tau_{I}}^{2} a}{a}=-\frac{2-\epsilon_{1}}{\tau_{I}^{2}\left(1-\epsilon_{1}\right)^{2}} .
\end{gathered}
$$

We can find that the breaking size $\theta_{I}$ becomes exactly equal to zero

$$
\theta_{I}=m_{I}^{2}+\frac{\tau_{I}}{2} \partial_{\tau_{I}} m_{I}^{2}=0
$$

Thus, for the power-law inflation model, the free actions preserve the dilatation symmetry. The same is true for the free scalar field on the exact de Sitter background. The asymptotic behavior of the mode function is governed by the growing mode

$$
\begin{aligned}
u_{I k} & \rightarrow A_{I} z_{I}+B_{I} z_{I} \int{ }^{\tau_{I}} \frac{d \tilde{\tau}_{I}}{z_{I}^{2}} \\
& \rightarrow A_{I} z_{I} \quad \text { when }\left|k \tau_{I}\right| \rightarrow 0,
\end{aligned}
$$

since

$$
\int \frac{\tau_{I}}{z_{I}^{2}} \tilde{\tau}_{I} \propto a^{-\left(3-\epsilon_{1}\right)}
$$

Consequently, we can obtain the asymptotic values of the spectral indices for the power-law inflation as

$$
n_{s}-\left.1\right|_{\left|k \tau_{I}\right| \rightarrow 0}=\left.n_{t}\right|_{\left|k \tau_{I}\right| \rightarrow 0}=-\frac{2 \epsilon_{1}}{1-\epsilon_{1}} .
$$

\section{Ultra slow roll inflation}

As another specific case, we consider the ultra slow roll inflation model. For this model, we cannot take the comoving gauge at the asymptotic future $\left|k \tau_{I}\right|=0$, but before that time, we can still work on the comoving gauge. The coefficients in the free action are given by

$$
\begin{aligned}
\mathcal{F}_{s} & =\mathcal{G}_{s}=\epsilon_{1} \propto a^{-6}, \\
\mathcal{F}_{t} & =\mathcal{G}_{t}=1 .
\end{aligned}
$$

In this model, the slow roll parameter $\epsilon_{1}$ rapidly decays in -6 powers of the scale factor, and thus other slow roll parameters become

$$
\begin{aligned}
f_{s 1} & =g_{s 1}=\epsilon_{2}=-6, \\
\text { others } & =0 .
\end{aligned}
$$

The mass of $u_{I}$ and the breaking sizes become

$$
\begin{aligned}
m_{s}^{2} & =-a^{2} H^{2}\left(2+2 \epsilon_{1}\right), \\
m_{t}^{2} & =-a^{2} H^{2}\left(2-\epsilon_{1}\right), \\
\theta_{s} & =-a^{2} H^{2}\left[8 \epsilon_{1}+2 \epsilon_{1}^{2}-\left(2-6 \epsilon_{1}+2 \epsilon_{1}^{2}\right) \sum_{i=1}^{\infty} \delta_{s i}\right] \\
& =O\left(a^{-4}\right), \\
\theta_{t} & =-a^{2} H^{2}\left[-\epsilon_{1}+\epsilon_{1}^{2}-\left(2-2 \epsilon_{1}^{2}\right) \sum_{i=1}^{\infty} \delta_{t i}\right]=O\left(a^{-4}\right) .
\end{aligned}
$$

Note that the summation of $\delta_{I i}$ starts from $i=1$, and all of $\delta_{I i \geq 1}$ is proportional to $\epsilon_{1}$ for this model. If we ignore all of the products proportional to $\epsilon_{1}$, we can omit the breaking term $\theta_{I}$ or $\Theta_{I}$. It is well known that for this model, the scalar mode function on the superhorizon scales is dominated by the decaying mode

$$
u_{s k} \rightarrow B_{s} z_{s} \int^{\tau_{s}} \frac{d \tilde{\tau}_{s}}{z_{s}^{2}} \quad \text { when } 0<\left|k \tau_{I}\right| \ll 1,
$$

since the decaying mode actually grows faster than the growing mode on the superhorizon scales

$$
\int^{\tau_{s}} \frac{d \tilde{\tau}_{s}}{z_{s}^{2}}=\int^{n} \frac{d \tilde{n}}{a^{3} H \mathcal{G}_{s}} \simeq \frac{1}{3 a^{3} H \mathcal{G}_{s}} \propto O\left(a^{3}\right) .
$$

The tensor mode function is dominated by the growing mode as usual. Using Eq. (42), we obtain the spectral indices on the superhorizon scales for the ultra slow roll inflation as 


$$
\begin{aligned}
n_{s}-\left.1\right|_{\left|k \tau_{I}\right| \ll 1} \simeq 2+2 \tau_{s}\left(\frac{\partial_{\tau_{s}} z_{s}}{z_{s}}+\frac{1}{z_{s}^{2} \int \tau_{s} \frac{d \tilde{\tau}_{s}}{z_{s}^{2}}}\right) \simeq 0, \\
\left.n_{t}\right|_{\left|k \tau_{I}\right| \ll 1} \simeq 2+2 \tau_{t}\left(\frac{\partial_{\tau_{t}} z_{t}}{z_{t}}\right) \simeq 0,
\end{aligned}
$$

where we ignore $O\left(\epsilon_{1}\right)$ terms since they rapidly decay into zero. While the horizon crossing formalism in the literature cannot estimate the scalar spectral index for the ultra slow roll inflation, our expression can predict it correctly since our expression is exact and can be applied to the generic single field models.
[1] A. H. Guth, Phys. Rev. D 23, 347 (1981).

[2] A. A. Starobinsky, Phys. Lett. 91B, 99 (1980).

[3] K. Sato, Mon. Not. R. Astron. Soc. 195, 467 (1981).

[4] P. A. R. Ade et al. (Planck Collaboration), Astron. Astrophys. 594, A20 (2016).

[5] Y. Akrami, J. Socorro, and R. Hernández-Jiménez, Astrophys. Space Sci. 364, 69 (2019).

[6] V. F. Mukhanov and G. V. Chibisov, Pis'ma Zh. Eksp. Teor. Fiz. 33, 549 (1981) [JETP Lett. 33, 532 (1981)].

[7] A. A. Starobinsky, Phys. Lett. 117B, 175 (1982).

[8] S. W. Hawking, Phys. Lett. 115B, 295 (1982).

[9] A. H. Guth and S. Y. Pi, Phys. Rev. Lett. 49, 1110 (1982).

[10] C. L. Bennett et al., Astrophys. J. 464, L1 (1996).

[11] E. Komatsu et al. (WMAP Collaboration), Astrophys. J. Suppl. 192, 18 (2011).

[12] P. A. R. Ade et al. (BICEP2 and Keck Array Collaborations), Phys. Rev. Lett. 121, 221301 (2018).

[13] A. Albrecht and P. J. Steinhardt, Phys. Rev. Lett. 48, 1220 (1982).

[14] A. D. Linde, Phys. Lett. 129B, 177 (1983).

[15] P. J. Steinhardt and M. S. Turner, Phys. Rev. D 29, 2162 (1984).

[16] J. Martin, C. Ringeval, and V. Vennin, Phys. Dark Universe 5-6, 75 (2014).

[17] C. Armendariz-Picon, T. Damour, and V. F. Mukhanov, Phys. Lett. B 458, 209 (1999).

[18] J. Garriga and V.F. Mukhanov, Phys. Lett. B 458, 219 (1999).

[19] T. Kobayashi, M. Yamaguchi, and J. Yokoyama, Phys. Rev. Lett. 105, 231302 (2010).

[20] N. Arkani-Hamed, P. Creminelli, S. Mukohyama, and M. Zaldarriaga, J. Cosmol. Astropart. Phys. 04 (2004) 001.

[21] M. Alishahiha, E. Silverstein, and D. Tong, Phys. Rev. D 70, 123505 (2004).

[22] C. Burrage, C. de Rham, D. Seery, and A. J. Tolley, J. Cosmol. Astropart. Phys. 01 (2011) 014.

[23] J. O. Gong and E. D. Stewart, Phys. Lett. B 510, 1 (2001).

[24] S. Habib, K. Heitmann, G. Jungman, and C. Molina-Paris, Phys. Rev. Lett. 89, 281301 (2002).

[25] J. Martin and D. J. Schwarz, Phys. Rev. D 67, 083512 (2003).

[26] R. Casadio, F. Finelli, A. Kamenshchik, M. Luzzi, and G. Venturi, J. Cosmol. Astropart. Phys. 04 (2006) 011.

[27] A. A. Starobinsky, Pis'ma Zh. Eksp. Teor. Fiz. 55, 477 (1992) [JETP Lett. 55, 489 (1992)].
[28] S. M. Leach and A. R. Liddle, Phys. Rev. D 63, 043508 (2001).

[29] J. A. Adams, B. Cresswell, and R. Easther, Phys. Rev. D 64, 123514 (2001).

[30] A. Makarov, Phys. Rev. D 72, 083517 (2005).

[31] A. A. Starobinsky, Pis'ma Zh. Eksp. Teor. Fiz. 42, 124 (1985) [JETP Lett. 42, 152 (1985)].

[32] M. Sasaki and E. D. Stewart, Prog. Theor. Phys. 95, 71 (1996).

[33] D. Wands, K. A. Malik, D. H. Lyth, and A. R. Liddle, Phys. Rev. D 62, 043527 (2000).

[34] D. H. Lyth, K. A. Malik, and M. Sasaki, J. Cosmol. Astropart. Phys. 05 (2005) 004.

[35] Y. i. Takamizu, arXiv:1804.07516.

[36] S. Weinberg, Phys. Rev. D 67, 123504 (2003).

[37] D. Baumann and D. Green, J. Cosmol. Astropart. Phys. 09 (2011) 014.

[38] V. Assassi, D. Baumann, and D. Green, J. Cosmol. Astropart. Phys. 11 (2012) 047.

[39] W. D. Goldberger, L. Hui, and A. Nicolis, Phys. Rev. D 87, 103520 (2013).

[40] K. Hinterbichler, L. Hui, and J. Khoury, J. Cosmol. Astropart. Phys. 01 (2014) 039.

[41] T. Tanaka and Y. Urakawa, J. High Energy Phys. 10 (2017) 127.

[42] D. Roest, J. Cosmol. Astropart. Phys. 01 (2014) 007.

[43] J. Martin, C. Ringeval, and V. Vennin, Phys. Rev. D 94, 123521 (2016).

[44] F. Lucchin and S. Matarrese, Phys. Rev. D 32, 1316 (1985).

[45] W. H. Kinney, Phys. Rev. D 72, 023515 (2005).

[46] T. Tanaka and Y. Urakawa, Prog. Theor. Exp. Phys. 2013, 083E01 (2013).

[47] R. Saitou, Phys. Rev. D 97, 083511 (2018).

[48] T. Kobayashi, M. Yamaguchi, and J. Yokoyama, Prog. Theor. Phys. 126, 511 (2011).

[49] G. W. Horndeski, Int. J. Theor. Phys. 10, 363 (1974).

[50] J. Gleyzes, D. Langlois, F. Piazza, and F. Vernizzi, J. Cosmol. Astropart. Phys. 08 (2013) 025.

[51] J. Gleyzes, D. Langlois, F. Piazza, and F. Vernizzi, Phys. Rev. Lett. 114, 211101 (2015).

[52] J. Gleyzes, D. Langlois, F. Piazza, and F. Vernizzi, J. Cosmol. Astropart. Phys. 02 (2015) 018.

[53] J. Gleyzes, D. Langlois, and F. Vernizzi, Int. J. Mod. Phys. D 23, 1443010 (2014).

[54] D. Langlois, M. Mancarella, K. Noui, and F. Vernizzi, J. Cosmol. Astropart. Phys. 05 (2017) 033. 